\title{
Multiwavelength Absolute Magnitudes and Colors of Red Clump Stars in the Gaia Era
}

\author{
Olcay Plevne ${ }^{1}$ (i), Özgecan Önal Taş ${ }^{2}$ (1) , Selçuk Bilir ${ }^{2}$ (1) , and George M. Seabroke ${ }^{3}$ \\ ${ }^{1}$ Istanbul University, Graduate School of Science, Department of Astronomy and Space Sciences, 34119, Beyazit, Istanbul, Turkey; olcayplevne@istanbul.edu.tr \\ ${ }^{2}$ Istanbul University, Faculty of Science, Department of Astronomy and Space Sciences 34119, Beyazit, Istanbul, Turkey \\ ${ }^{3}$ Mullard Space Science Laboratory, University College London, Holmbury St Mary, Dorking, RH5 6NT, UK \\ Received 2019 December 6; revised 2020 March 10; accepted 2020 March 16; published 2020 April 21
}

\begin{abstract}
This study presents the multiwavelength investigation of the absolute magnitudes and colors of the red clump (RC) stars selected from APOGEE and GALAH DR2 combined catalog which is complemented with Gaia DR2 astrometric data and multiwavelength photometric data of GALEX GR6/7, SDSS DR7, Gaia DR2, 2MASS, and WISE sky surveys. The analyses are centered on the different distance estimation methods using Gaia trigonometric parallaxes, $(1 / \varpi)$ and Bayes statistics, and chemically defined Galactic disk populations on $[\alpha / \mathrm{Fe}] \times[\mathrm{Fe} / \mathrm{H}]$ plane. This investigation questions the long-studied problem of the population effects on $\mathrm{RC}$ luminosity. Using two different distance estimation approaches, (i) chemical thin and chemical thick disk RC stars are shown to have different absolute magnitudes, while colors remain the same in all photometric bands. Absolute magnitudes vary between -0.12 and $+0.13 \mathrm{mag}$ for the $1 / \varpi$ with the change of the Galactic population. This variation in absolute magnitudes is found to be larger for the other method, (ii) the Besançon population synthesis model of Galaxy for 2MASS photometry, in which the absolute magnitude difference between chemical populations were found between -0.35 and -0.40 mag from thin disk to thick disk. When results compared with each other, differences of absolute magnitudes are about three times larger in the model than in the observations. We confirm that the RC absolute magnitudes depend on $\alpha$-element abundances of Galactic populations.
\end{abstract}

Unified Astronomy Thesaurus concepts: Red giant clump (1370); Absolute magnitude (10); Stellar populations (1622)

\section{Introduction}

Galactic archeology studies require objects that are observable in a vast space volume, like the Gaiasphere, in order to construct formation and evolution scenarios that lead to the present day picture from the past events of the Milky Way galaxy. The critical parameter for these studies is reliably estimated distance, which is the current hot topic. As of the start of the Gaia era (Gaia Collaboration et al. 2016), our blurred vision of Galactic structure is become more clear starting from the Solar neighborhood with the support of the advanced computer programming abilities that grow within the scientific community. The second data release of Gaia (Gaia Collaboration et al. 2018) contains the largest data set with the most accurate 5D astrometric data, superseding its predecessor, the Hipparcos astrometry satellite (Perryman et al. 1997), by combining spectroscopic and astrometric data allows to obtain the most accurate 12D parameter space for Galactic evolution studies. The 25 yr gap between Hipparcos and Gaia was filled with developing and/or improving alternative distance estimation methods via photometric and/or spectroscopic properties of various celestial objects for different distance ranges in the universe, called the distance ladder. Cepheid variables and $\mathrm{SNe}$ Ia are considered as "standard candles" due to their absolute magnitudes at a certain evolutionary stage at which they radiate precise energy output. One other object is alternatively considered to be a standard candle in the last $40 \mathrm{yr}$ : red clump stars.

Red clump (RC) stars, which are visible throughout great distances, such as neighboring galaxies, and their almost constant brightness gives them potential standard candle status. All the data regarding their standard candle status were obtained from spectroscopic survey data with spectrophotometric distances before the Gaia era.
Cannon \& Lloyd (1969) showed that it is possible to determine clumps of red giant stars from their color and surface gravity properties and their distances can also be calculated using photometric data. Investigating the red giant stars in Hertzsprung-Russell (HR) diagrams of rich and old open clusters, Cannon (1970) pointed out that these red giant stars are clumped around $\left(M_{V}, B-V\right)=(1,1)$ mag on the colormagnitude diagram. Then, based on the stellar interior models of the time (Faulkner 1966; Iben 1967), the possible cause of this clumping is examined and we arrived the conclusion that these are the post-helium flash stars with stable helium burning inside their cores and their masses are less than $2.25 \mathrm{M}_{\odot}$. According to theory, RCs are metal-rich, low-mass stars $\left(M<2.25 M_{\odot}\right)$ with stable helium burning cores, which cause a narrow range of luminosity. Since their first recognition by Cannon (1970), there have been numerous debates on intrinsic properties, populations, and absolute magnitudes of RC stars in different parts of the electromagnetic spectrum, i.e., ultraviolet (UV), optical, near-infrared (NIR), and mid-infrared (MIR).

A significant contribution to $\mathrm{RC}$ studies started with the more precise parallax measurements from Hipparcos mission (Perryman et al. 1998). The RC region was a very prominent feature on the color-magnitude diagram of the Solar neighborhood stars observed by Hipparcos. The Hipparcos catalog (ESA 1997) contained approximately 600 RC stars with relative parallax errors less than 0.1 (Girardi et al. 1998). These precise parallax measurements triggered extensive studies on the dependencies of the RC absolute magnitude to age and chemistry. By doing so, the absolute magnitudes of RC stars selected from different regions of the Milky Way and neighboring galaxies were calibrated many times. First studies used optical photometric bands $V$ and $I$. Metallicity dependence of the $I$-band magnitudes were investigated by several authors 
in the literature (Paczyński \& Stanek 1998; Perryman et al. 1998; Udalski 2000), and it is found to be weak for this region. Moreover, Sarajedini (1999) and Twarog et al. (1999) claimed the RC magnitudes depend on the metallicity and age by using RCs in open clusters, which is supported by the later models (Girardi \& Salaris 2001; Salaris \& Girardi 2002). Using Hipparcos stars, Alves (2000) made a calibration for 2MASS $K_{s}$-band (Cutri et al. 2003), which is less affected by reddening and mild systematic dependence on metallicity. Under the assumption of no reddening, Alves (2000) found $M_{K_{s}}=$ $-1.61 \pm 0.03 \mathrm{mag}$, with a linear relation with metallicity, i.e., $M_{K_{s}}=0.57(0.36) \times[\mathrm{Fe} / \mathrm{H}]-1.64(0.07)$.

The real leap on RC studies occurred when the re-reduced Hipparcos data (van Leeuwen 2007), in which the parallax measurements and their respective errors were updated. Groenewegen (2008) calculated the absolute magnitudes for a sample selected from re-reduced Hipparcos catalog and found mean absolute magnitudes in optical $I$ and NIR $K_{s}$ bands as $\left\langle M_{I}\right\rangle=-0.22 \pm 0.03 \mathrm{mag}$ and $\left\langle M_{K_{s}}\right\rangle=-1.54 \pm 0.04 \mathrm{mag}$, respectively. Also, Groenewegen (2008) modeled a synthetic $\mathrm{RC}$ star sample by applying a number of selection criteria on metallicity, age, magnitude range, etc. in order to infer the effects of population and absolute magnitude selection. These analyses showed that the $M_{I}$ and $M_{K_{s}}$ are weakly related to metallicity and $V-K$ color, respectively. As the wide-area NIR all-sky surveys 2MASS, DENIS, became more complete, the RC studies to validate the standard candle status are spread out to the NIR photometric bands. According to Salaris (2013), $K_{s}$-band is the ideal photometric band to determine RC distances, because the star formation history does not change in this band. In Bilir et al. (2013b), metallicity dependence of $\mathrm{RC}$ stars are selected from open and globular clusters in optical bands. The first absolute magnitude determination in WISE photometric bands was performed by Yaz Gökçe et al. (2013)'s large RC sample, and there have been numerous studies (Chen et al. 2017; Ruiz-Dern et al. 2018). In a recent study, Mohammed et al. (2019) analyzed APOGEE DR14 RC stars in GALEX NUV band with Gaia $G$ band, and found a strong dependence of color on effective temperature and metallicity.

Chemical abundances of stars changes drastically in Galaxywide distances so that various metallicity gradients were obtained in various radial and vertical directions from the Galactic center and the Galactic plane, respectively (Coşkunoğlu et al. 2012; Plevne et al. 2015; Önal Taş et al. 2016, 2018; Tunçel Güçtekin et al. 2019). Up until the Gaia era, our RC knowledge was gathered from the stars within the Solar neighborhood with different radii $(5<R<10 \mathrm{kpc}$ and $-2<|Z|<2 \mathrm{kpc}$ ). Nowadays, thanks to the precise astrometry of Gaia DR2 (Gaia Collaboration et al. 2018) and the high resolution $(R>22,000)$ and high signal-to-noise $(\mathrm{S} / \mathrm{N}>100)$ of APOGEE (Majewski et al. 2017) and GALAH DR2 (Buder et al. 2018) spectroscopy, RC stars have become appealing objects to study.

Many studies in the last $25 \mathrm{yr}$ established various median absolute magnitudes in optical, NIR, and MIR parts of the electromagnetic spectrum (Girardi 2016). However, these studies were biased toward the bright nearby objects within the Solar neighborhood due to sample selection using relative parallax errors to obtain dependable subsamples. According to the review study of Girardi (2016), RC stars are more abundant than horizontal branch stars. Thus, these stars are one of the main targets of brightness-limited sky surveys. Based on their spectral types (G8-K2), they are ideal objects for accurate radial velocity and chemical abundance determination (Saguner et al. 2011).

The era of wide-area imaging surveys such as GALEX (Martin et al. 2005), SDSS (Abazajian et al. 2004), 2MASS (Skrutskie et al. 2006), and WISE (Wright et al. 2010), allow probing the multiwavelength properties (from UV to MIR) of any selected stellar population. The combination of these surveys with the precise astrometric properties of Gaia DR2 (Gaia Collaboration et al. 2018) and the high resolution and high $\mathrm{S} / \mathrm{N}$ of spectroscopic surveys like APOGEE (Allende Prieto et al. 2008) and GALAH (de Silva et al. 2016), in particular, allow an in depth study.

This study is focused on the multiwavelength absolute magnitudes and colors of RC stars in low- and high- $[\alpha / \mathrm{Fe}]$ populations in the Galactic disk. The study also deals with different distance estimation methods. The paper is organized as follows. Data selection and RC identification are described in Section 2, distance estimation methods, interstellar reddening, and chemical separation of RC stars are presented in Section 3, and results of the multiwavelength absolute magnitudes and colors are given in Section 4. Results of a mock catalog are given in Section 5, RC contamination and the effects of the absolute magnitudes on estimated distances are given in Section 6, and summary and conclusions are given in Section 7.

\section{Data}

\subsection{Red Clump Star Selection}

In this study, we use spectroscopic data provided by SDSSIV DR14 APOGEE (Majewski et al. 2017) and GALAH DR2 (Buder et al. 2018) surveys. Both surveys have similarities such as high resolution, high $\mathrm{S} / \mathrm{N}$, spectral analysis pipeline The Cannon (Ness et al. 2015). The only difference between these surveys is their observation grounds, one is made from the Apache Point Observatory in the Northern hemisphere, and the other is made at the Anglo Australian Observatory in the Southern hemisphere. Preliminary data selection is made by eliminating the samples from the objects with low $\mathrm{S} / \mathrm{N}$ spectra. Then, stars with missing $T_{\text {eff }}, \log g,[\mathrm{Fe} / \mathrm{H}],[\alpha / \mathrm{Fe}]$, and radial velocity data are also eliminated. Moreover, selection flagcan$n o n=0$ and observation with the highest $\mathrm{S} / \mathrm{N}$ are selected. As a result of these cuts, 154,801 stars in APOGEE and 188,750 stars in GALAH DR2 are found. By combining both catalogs, a master catalog of 343,551 stars is obtained. This catalog is named APOGEE-GALAH Red Clump (AGRC) catalog. Spectroscopic HR diagrams of these stars that are plotted by stellar number density $(\log N)$ and metallicity $([\mathrm{Fe} / \mathrm{H}])$ are shown in Figure 1. There are three more populated regions on the diagram, which correspond to main-sequence (MS), red giant branch (RGB), and red clump (RC) stars. Effective temperature of stars varies between $3500 \leqslant T_{\text {eff }}(\mathrm{K}) \leqslant 7000$ and their metallicity varies between $-3 \leqslant[\mathrm{Fe} / \mathrm{H}](\mathrm{dex}) \leqslant$ +0.5 . In order to separate $\mathrm{MS}, \mathrm{RGB}$, and $\mathrm{RC}$ regions, three Gaussian functions are fitted on the stellar number density distribution of each region as shown in Figure 2. According to the Gaussian fits, MS population covers $4500<$ $T_{\text {eff }}(\mathrm{K})<7000$ and $3.6<\log g(\mathrm{cgs})<4.8$, RGB population covers $3500<T_{\text {eff }}(\mathrm{K})<5300$ and $0<\log g(\mathrm{cgs})<3.8$, and RC population covers $4500<T_{\text {eff }}(\mathrm{K})<5200$ and $2.1<$ $\log g$ (cgs) $<2.7$ on the HR diagram. The most likely region 

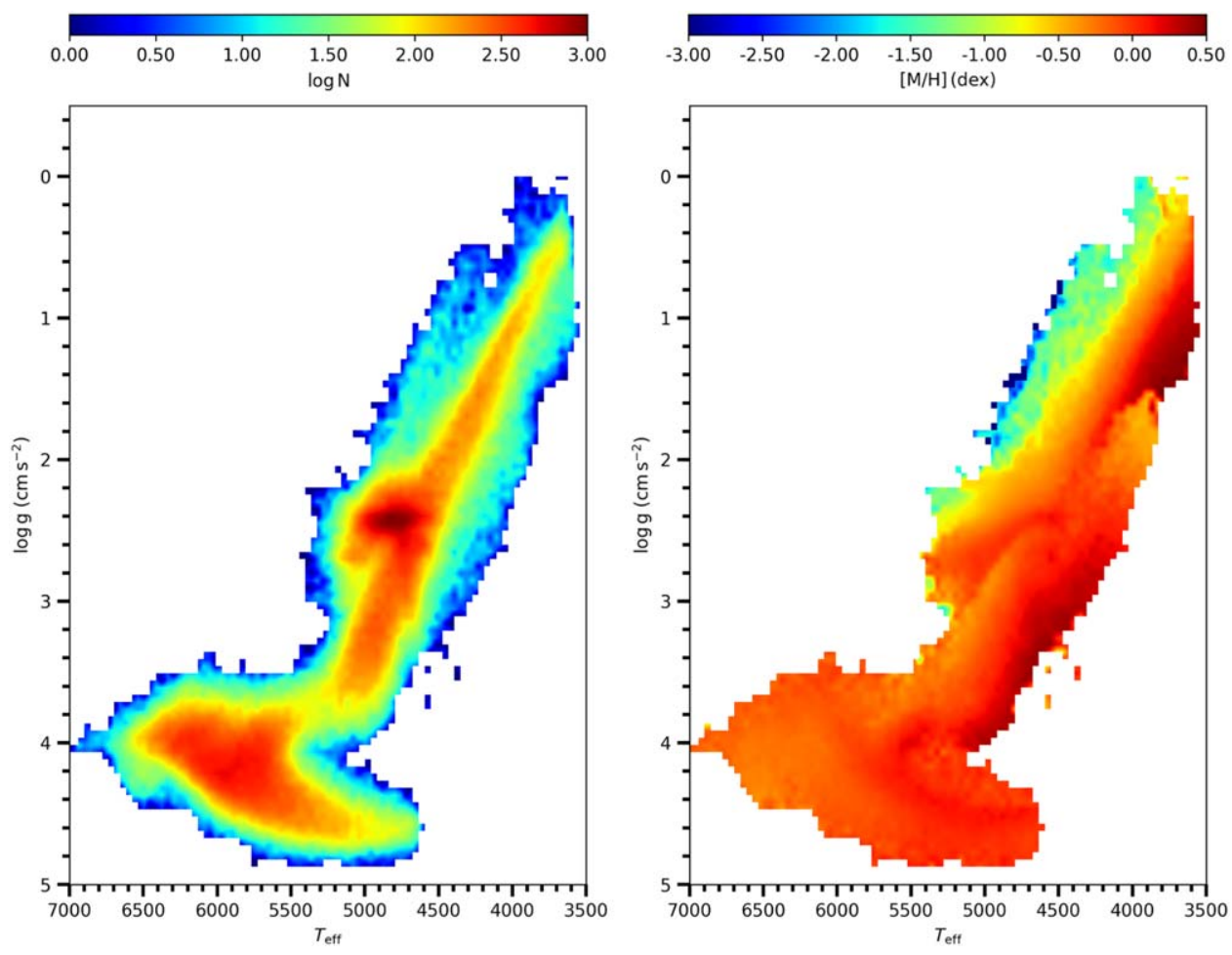

Figure 1. HR diagrams of the APOGEE and GALAH DR2 combined catalog stars, which are color coded for the stellar number density (left panel) and the metallicity (right panel), respectively.

where RC population resides on the HR diagram has central coordinates $\left(T_{\text {eff }}, \log g\right)=(4834 \pm 190 \mathrm{~K}, 2.43 \pm 0.105 \mathrm{cgs})$, and standard deviations are calculated with the width of onehalf maximum of the Gaussian distributions, i.e., FWHM = $2.35 \times \sigma$. The RC population is defined by selecting the $2 \sigma$ region around these central coordinates, which is shown as a black short dashed ellipse on the HR diagram. There are 47,537 stars inside this ellipse.

Spectroscopic data are complemented with astrometric data from Gaia DR2. Quality of trigonometric parallaxes are determined by calculating the relative parallax errors $\left(\sigma_{\varpi} / \varpi\right)$ of the sample stars. There exists a systematic scatter in trigonometric parallax measurements, according to Lutz \& Kelker (1973). Trigonometric parallaxes are affected by bias so that the obtained distances scatter as the observed volume increase. Bias correction function is introduced by Smith (1987)'s study, and further studied for Hipparcos mission by Oudmaijer et al. (2002). Based on the comparative analysis between ground- and space-based parallax measurements with relative parallax errors, it is shown that there is a limiting value of $\sigma_{\varpi} / \varpi=0.175$. This is defined as the upper limit to correct parallaxes for LK bias.

Relative parallax distribution of AGRC catalog is given in Figure 3. The relative parallax error distribution ranges up to 0.6 and the median value of AGRC sample is 0.08 . In this study, the relative parallax error limit is selected as 0.1 because we wanted to include stars from different Galactic populations in the AGRC sample. The final sample of AGRC catalog is 23,880 . The AGRC catalog is separated into relative parallax error subsample intervals at $0.05,0.08$, and 0.10 . These intervals include $35 \%, 45 \%$, and $20 \%$, respectively, of the AGRC sample. For these subsamples, the LK corrections to be applied to the Gaia parallaxes using Equation (12) of Smith (1987) were calculated as $\leqslant 1 \%, 1 \%-2.6 \%$, and $2.6 \%-4.2 \%$, respectively. We decided to not to apply LK correction to the AGRC catalog (see also, Çelebi et al. 2019).

\section{Methods}

\subsection{RC Distances}

$\mathrm{RC}$ distances are estimated using two separate methods. One is the conventional inverse parallax $(1 / \varpi)$ method, in which the distances are directly calculated from Gaia DR2 trigonometric parallaxes (Gaia Collaboration et al. 2018). The other is the probabilistic analysis using priors like trigonometric parallaxes and their uncertainties to estimate the source distances by considering the variations based on the Galactic coordinates and scale height of the disk in a model of the Galaxy (Bailer-Jones 2015; Bailer-Jones et al. 2018); we call it the BJ18 method. As it is pointed out by the author, the observational errors in parallaxes can produce bias in samples. This bias is especially effective when the trigonometric parallax values are either negative or their relative errors are larger than 0.2 .

Comparison between stellar distances with $1 / \varpi$ and BJ18 methods are shown in Figure 4. Data points are colored based on the relative parallax errors. The increase in percentages of the AGRC stars along with the distance is given in the upper panel, while distance residuals are given in the lower panel. The AGRC catalog covers the stars within $7 \mathrm{kpc}$ distance and $80 \%$ of the sample lies within less than $3 \mathrm{kpc}$ distance, according to Figure 4. Distances start to deviate from each other at $1 \mathrm{kpc}$ and this becomes apparent at $2 \mathrm{kpc}$. The distance difference becomes larger with increasing distance and reaches $1 \mathrm{kpc}$ at $6 \mathrm{kpc}$ distance in $1 / \varpi$ scale. In this figure, relative parallax errors of RC stars are color coded, and relative parallax errors increase with the distance. This study implies that the 


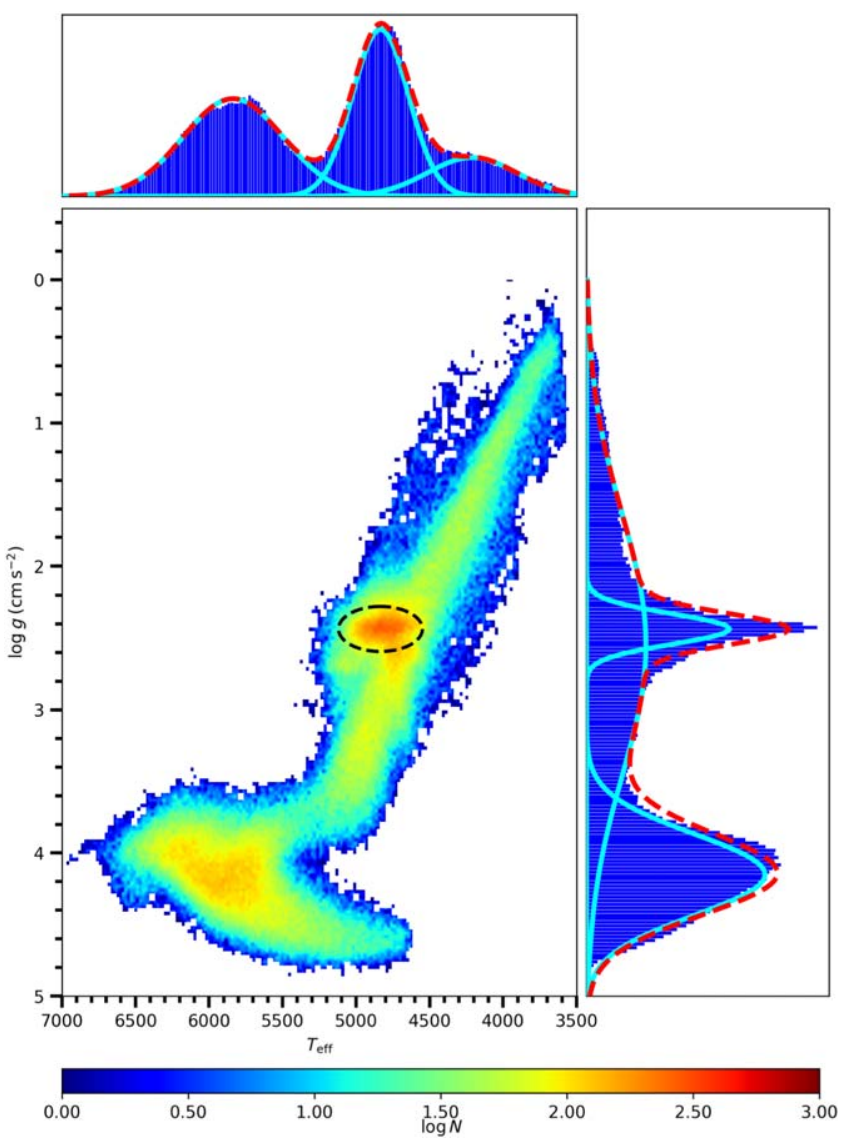

Figure 2. Separation of different luminosity classes and selection of the RC stars on HR diagram. Middle panel shows the Kiel diagram of all stars. Upper and right panels give the frequency distributions of $T_{\text {eff }}$ and $\log g$. Red dashed lines give the overall distribution of stars in each parameter. Turquoise solid lines give the distribution of each luminosity class embedded in the overall distribution.

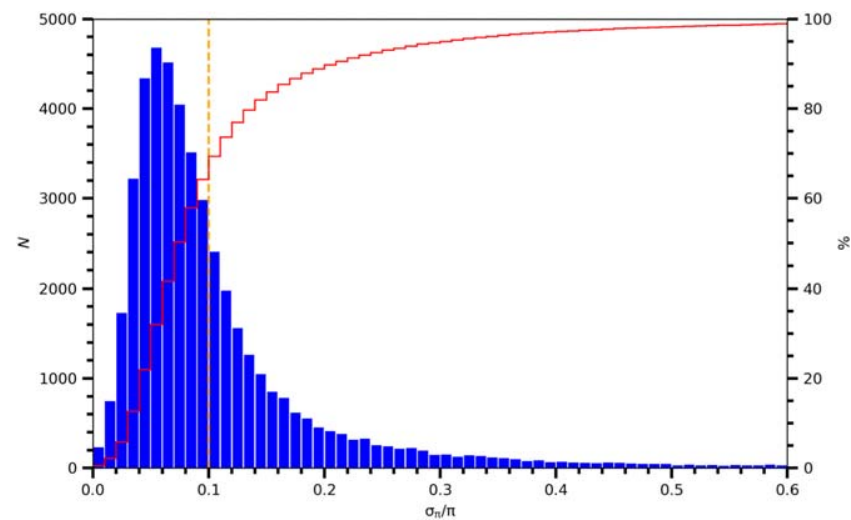

Figure 3. Relative parallax error distribution of the AGRC stars. Step function is gives the increase of percentage of the AGRC stars with increasing relative parallax errors. Yellow dashed line shows the limit of $\sigma_{\varpi} / \varpi=0.1$.

distance estimation method is not a critical ingredient up to $2 \mathrm{kpc}$.

Heliocentric coordinates of AGRC stars are calculated using their Galactic coordinates and distances. Heliocentric distance distributions of RC stars on $X-Y$ (left panels) and $X-Z$ (right panels) planes only for $1 / \varpi$ method is given in Figure 5 . In the upper panels, red and blue circles denote APOGEE and GALAH DR2 surveys, respectively. In the lower panels, the $\mathrm{RC}$ stars are shown in logarithmic number density. Median

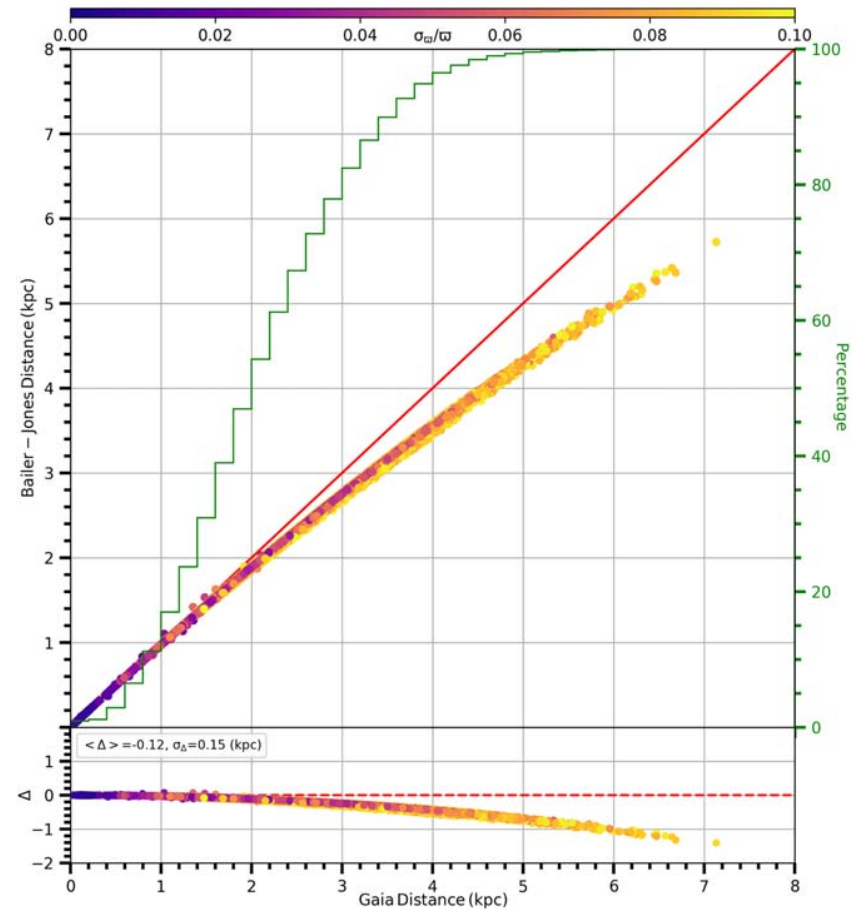

Figure 4. Distance comparison between $1 / \varpi$ and BJ18 methods using AGRC catalog. Stars are represented with colors of individual relative parallax errors. Also, on the right $y$-axis, the increment of the sample with increasing distances is shown with a green step function. In the lower panel, the residual distances are shown with $\langle\Delta\rangle=0.12 \mathrm{kpc}$ and $\sigma_{\Delta}=0.15 \mathrm{kpc}$. Red dashed line represents the zero line.

distances to the Sun and median heliocentric distance components for APOGEE, GALAH DR2, and all sample are listed in Table 1.

\subsection{Chemical Separation of RC Stars with Unsupervised Machine Learning}

The Galactic disk, as a dynamically evolving object, has regions that are subjected to different internal and external dynamics and chemical processes during its 13 Gyr evolution. It is well known that kinematic properties of stars can be altered, especially in the presence of a dominant angular momentum redistributor (or perturber) such as Galactic bar, spiral arms, and giant molecular clouds or even streams of stars. However, chemistry evolves differently in the course of time, depending on the initial mass of stars. Trends in chemistry are suggested by the Freeman \& Bland-Hawthorn (2002) study, indicating that stars keep memory of the chemical structure of their birth cluster. Chemical characteristics have been proposed as a way to parameterize the properties of various stellar populations in the Milky Way (scale height, scale length, kinematics, see Bovy et al. 2012). Throughout the Galactic disk, the abundance ratios $([\mathrm{Fe} / \mathrm{H}],[\alpha / \mathrm{Fe}]$ etc. $)$ of stellar samples are known to change toward the radial direction from the Galactic center or in the vertical direction from the Galactic plane (Bilir et al. 2006, 2008, 2012; Cabrera-Lavers et al. 2007a). There is strong evidence that there are two main structures embedded in the Galactic disk with clearly distinct, but partly overlapping properties, which are known as thin disk and thick disk (Gilmore \& Wyse 1985; Gratton et al. 1996; Fuhrmann 1998, 2008; Prochaska et al. 2000; Bensby et al. 2003, 2007; Reddy et al. 2006; Navarro et al. 2011; Haywood et al. 2019; 

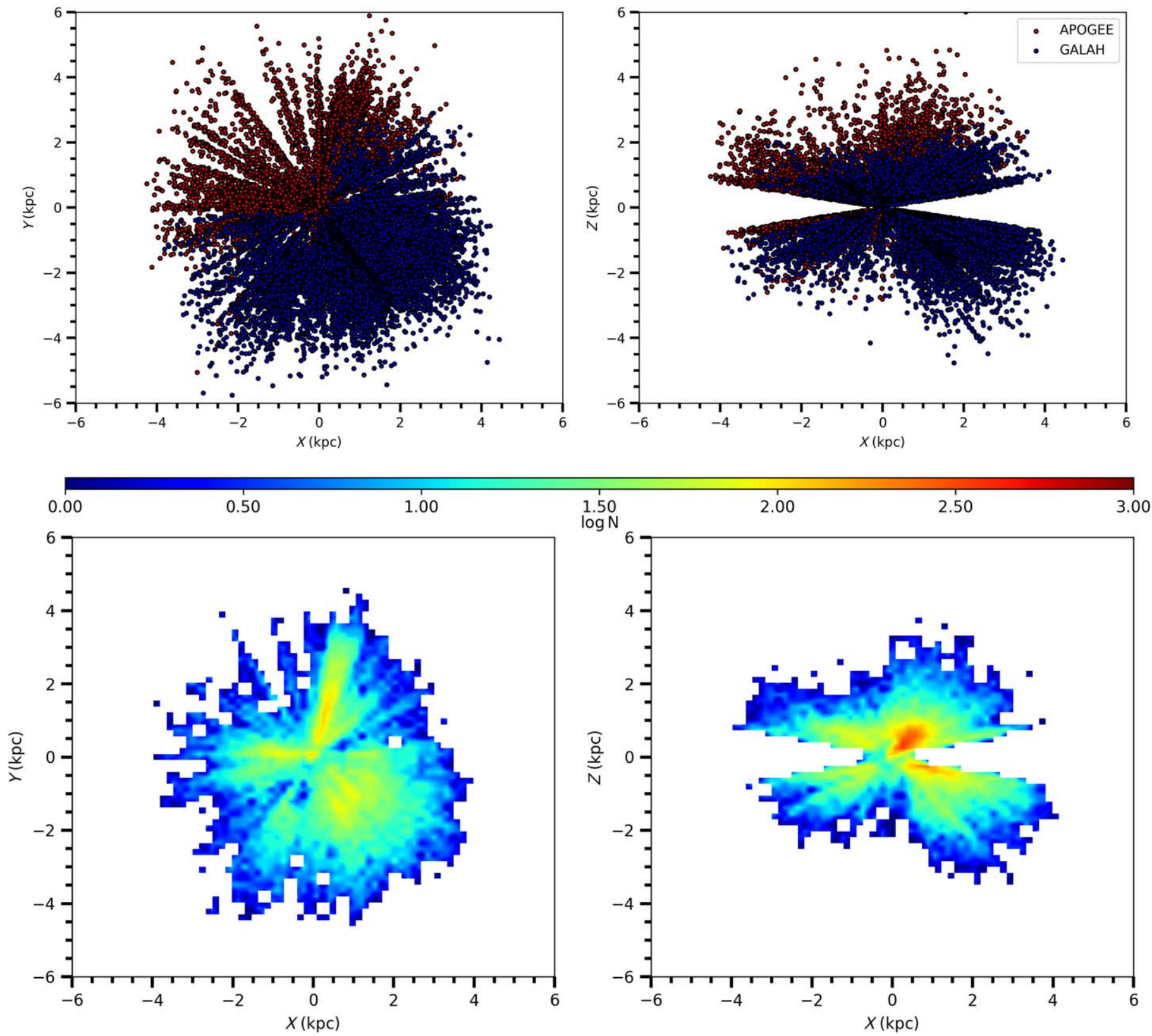

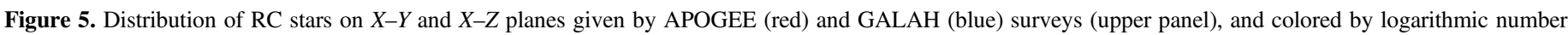
density (lower panel). The Galactic center is at approximately $+8 \mathrm{kpc}$ on the $x$-axis (Majewski 1993).

Karaali et al. 2019). Thin disk stars are younger, rich in $[\mathrm{Fe} / \mathrm{H}]$, poor in $[\alpha / \mathrm{Fe}]$, and have cold kinematic properties, while thick disk stars are significantly older, poor in $[\mathrm{Fe} / \mathrm{H}]$, rich in $[\alpha / \mathrm{Fe}]$, and have hot kinematic properties. Distribution of stars on this plane is a reflection of the chemical evolution of the Milky Way. Investigations of the chemical evolution of the Galactic disk have shown that the disk has undergone at least two different periods of formation Chiappini et al. (1997). The stars that are born during these formation periods can be separated on $[\alpha /$ $\mathrm{Fe}] \times[\mathrm{Fe} / \mathrm{H}]$ plane. It turns out that the chemical abundances plotted in this plane can be used to disentangle the components of the Galactic disk in age (Wyse \& Gilmore 1988). This discrete structure persists even at different radial distances along the Galactic disk (Haywood 2008). Although the disk consists of two separate chemical populations, different studies examining this distinction have not been able to establish a specific criterion for separation because populations are intertwined in the chemical plane.

This problem can be solved in our study by using the Gaussian Mixture Model (GMM), an unsupervised machinelearning algorithm, to separate our RC sample into chemical populations. This algorithm classifies the data by fitting the desired number of Gaussian planes to the data. Classification is made by calculating the probabilities of the data of each Gaussian plane. Also, the different number of Gaussian sets can be fitted using different statistical methods. However, in this study, there is no need to do this because previous observational findings and observational models indicate that the Galaxy disk is composed of two separate chemical populations (Freeman \& Bland-Hawthorn 2002), which are mentioned in Section 3.1. In this study, a machine-learning library sklearn version 0.19.1 (Pedregosa et al. 2011) is used to apply GMM to 

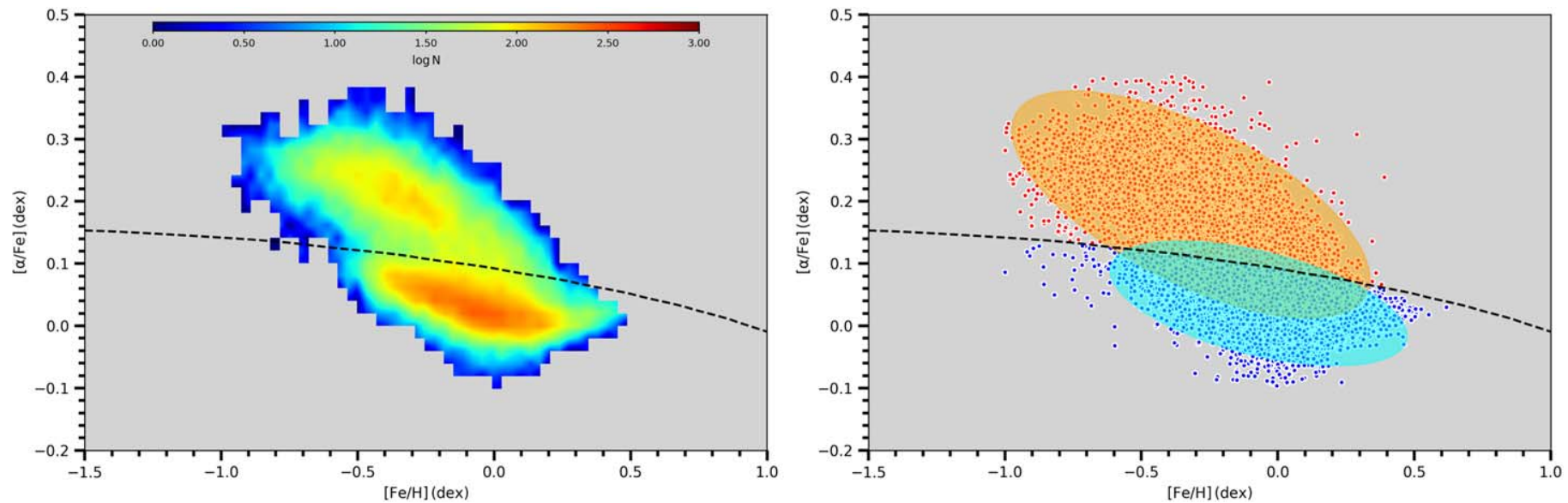

Figure 6. Distribution of $\mathrm{RC}$ stars on $[\alpha / \mathrm{Fe}] \times[\mathrm{Fe} / \mathrm{H}]$ plane. Left panel: the distribution is given for logarithmic number density. The black dashed line presents the decision boundary obtained with Gaussian mixture method. Right panel: a scatter diagram of RC stars is given with red and blue dots for high- $\alpha$ and low- $\alpha$ populations, respectively. Throughout the paper, red and blue colors will represent these populations. Orange and blue ellipses represent the $1 \sigma$ probability distribution of each Gaussian for chemical space.

Table 1

Distance Distribution of RC Stars for APOGEE, GALAH DR2, and all Stars for 1/๘ (Upper Panel) and BJ18 (Lower Panel) Methods

\begin{tabular}{|c|c|c|c|c|c|}
\hline & \multicolumn{5}{|c|}{$1 / \varpi$ Method } \\
\hline & $\tilde{d}(\mathrm{kpc})$ & $\tilde{X}(\mathrm{kpc})$ & $\tilde{Y}(\mathrm{kpc})$ & $\tilde{Z}(\mathrm{kpc})$ & $N$ \\
\hline APOGEE & $1.85 \pm 1.02$ & $0.11 \pm 1.34$ & $0.83 \pm 1.66$ & $0.56 \pm 1.04$ & 10,798 \\
\hline \multirow[t]{2}{*}{ All } & $2.08 \pm 1.02$ & $0.49 \pm 1.19$ & $-0.39 \pm 1.21$ & $0.26 \pm 0.87$ & 23,880 \\
\hline & \multicolumn{5}{|c|}{ BJ18 Method } \\
\hline GALAH & $2.21 \pm 0.99$ & $0.97 \pm 1.32$ & $-1.35 \pm 1.02$ & $-0.39 \pm 0.89$ & 13,082 \\
\hline All & $1.96 \pm 1.00$ & $0.49 \pm 1.22$ & $-0.39 \pm 1.52$ & $0.27 \pm 0.94$ & 23,880 \\
\hline
\end{tabular}

Note. Columns give sample, median values of distance, heliocentric $X, Y$, and $Z$ distances and number of stars.

the RC data. Model parameters are as follows: $n$ _components parameter, which specifies the number of entered classes, is set to 2 and covariance_type, which defines the covariance matrices of Gaussian surfaces while assigning each class, is selected as Full, because each plane is a separate covariance matrix and the planes intersect with each other. The AGRC sample is separated into two classes as expected from the GMM and results are shown in Figure 6. In the figure, the orange and blue ellipses represent the $1 \sigma$ probability distribution of the Gaussian planes determined by the model for two chemical populations, and the decision boundary is shown with a dashed black line. This boundary passes through the points where the possibilities of the two models are equal. As a result, GMM classified the region above this line as high- $[\alpha / \mathrm{Fe}]$ and below as low- $[\alpha / \mathrm{Fe}]$ (hereafter low- $\alpha$ and high- $\alpha$, respectively). According to GMM, there are 13,635 stars in low- $\alpha$, while 10,245 stars in high- $\alpha$ population.

\section{Absolute Magnitudes and Colors from UV to MIR}

In this study, stellar distances are estimated with $1 / \varpi$ and BJ18 methods using Gaia DR2 trigonometric parallaxes. Absolute magnitudes of stars are obtained via Pogson's relation by using stellar distances, apparent magnitudes from UV to MIR, and interstellar extinction related coefficients from various authors (Table 2). Then, the median value of absolute
Table 2

Coefficients of Interstellar Extinction for Each Photometric Band from Near UV to MIR Wavelengths

\begin{tabular}{lll}
\hline \hline Band & $A_{\lambda} / A_{V}$ & Reference \\
\hline$N U V$ & 2.335 & Yuan et al. (2013) \\
$u$ & 1.567 & An et al. (2009) \\
$g$ & 1.196 & An et al. (2009) \\
$r$ & 0.874 & An et al. (2009) \\
$i$ & 0.672 & An et al. (2009) \\
$z$ & 0.488 & An et al. (2009) \\
$G$ & 0.859 & Olivares et al. (2019) \\
$J$ & 0.887 & Fiorucci \& Munari (2003) \\
$H$ & 0.565 & Fiorucci \& Munari (2003) \\
$K_{s}$ & 0.382 & Fiorucci \& Munari (2003) \\
$W 1$ & 0.039 & Wang \& Chen (2019) \\
$W 2$ & 0.026 & Wang \& Chen (2019) \\
$W 3$ & 0.040 & Wang \& Chen (2019) \\
\hline
\end{tabular}

Note. Columns show photometric band, extinction coefficient and reference.

magnitude distribution is determined for each chemical population in each photometric band. Building on these simple absolute magnitude estimations from the observational data, the $\mathrm{RC}$ distances are calculated by assuming a single absolute magnitude value for each chemical population where the results 


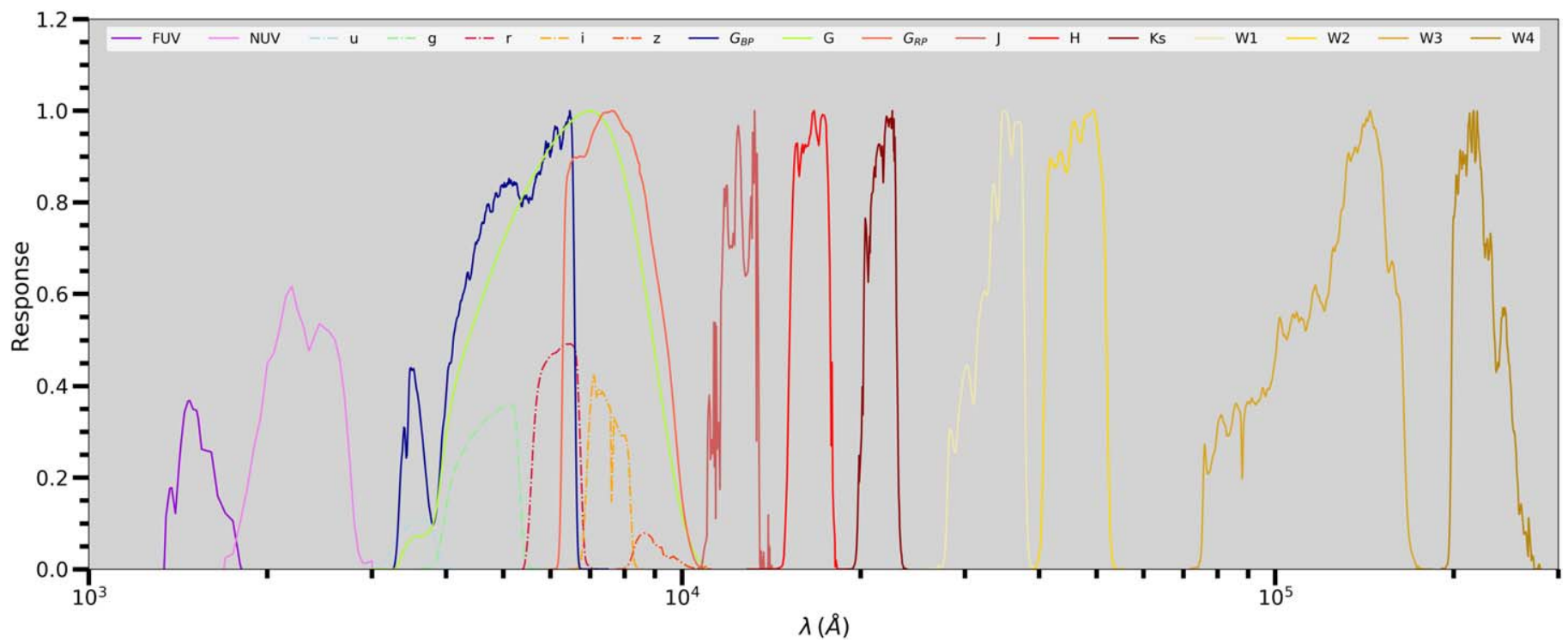

Figure 7. Response curves of photometric bands used in this study. Starting from left to right are GALEX FUV (magenta), NUV (pink), SDSS $u$ (dashed light blue), $g$ (dashed green), $r$ (dashed red), $i$ (dashed yellow), $z$ (dashed orange), Gaia $G_{B P}$ (dark blue), $G$ (green), $G_{R P}$ (red), 2 MASS $J$ (pale red), $H$ (bright red), $K_{S}$ (dark red), WISE $W 1$ (pale yellow), W2 (yellow), W3 (light brown), and W4 (brown) bands.

show deviation (i) as the population changes, (ii) as the photometric band changes, (iii) as the distance estimation method changes.

Since this study is aimed at investigating photometric distance determination for RC stars, it is important to perform an accurate estimate of the interstellar extinction and reddening. Galactic dust maps give extreme values at low Galactic latitudes, even though various methods reduce these values. Stars above the Galactic plane, $|b| \geqslant 10^{\circ}$, are selected in order to minimize the effect of interstellar extinction. The total extinction $A_{\infty}(b)$ in $V$-band for a star's direction is obtained from the dust map ${ }^{4}$ of Schlafly \& Finkbeiner (2011) and estimated extinction $A_{d}(b)$ for the distance between Sun and the star using the equation of Bahcall \& Soneira (1980) by adopting a scale height of Galactic dust as $H=125 \mathrm{pc}$ (Marshall et al. 2006). Details of the analysis used in estimating reduced extinction can be seen from Tunçel Güçtekin et al. (2016).

Absolute magnitudes of RC stars are calculated using the conventional relation between apparent magnitude and distance in the form of $M_{\lambda}=m_{\lambda}-5 \log d+5-A_{\lambda}$. Here $M_{\lambda}, m_{\lambda}, d$, and $A_{\lambda}$ are absolute magnitude, apparent magnitude, distance, and interstellar extinction in the selected photometric band, respectively. Interstellar extinction coefficients for each photometric band are given in Table 2. Absolute magnitudes of RC stars in UV, optical, NIR, and MIR of the electromagnetic spectrum are derived using data collected from GALEX GR6/7 (Bianchi et al. 2017), SDSS DR7 (Abazajian et al. 2009), Gaia DR2 (Gaia Collaboration et al. 2018), 2MASS (Cutri et al. 2003), and All-WISE (Cutri et al. 2013) surveys. Response curves of individual photometric bands of each photometric system are given in Figure 7 . In the study, the absolute magnitudes are determined by reading the median value of the band in question of the applied Gaussian fit on the frequency distribution in each individual wavelength range. Note that there are two main data sets, one is absolute magnitudes, and

\footnotetext{
4 https://irsa.ipac.caltech.edu/applications/DUST/
}

the other is colors. Each data set is further separated into lowand high- $\alpha$ populations with the method mentioned in Section 3.2, and then evaluated for the two distances that are obtained with different estimation methods. Overall results of median absolute magnitude and color determinations are given in Table 3 with consecutive panels. Each data set is evaluated specifically for the chosen surveys' unique conditions, i.e., quality flags, magnitude limits etc.; therefore subsamples vary for each photometric band. Thus, based on this approach, the number of sources for each photometric system is different and unique, except for WISE. The number of stars varies within each WISE band.

\subsection{Ultraviolet Region}

The UV region is covered by the data of the GALEX GR6/7 survey with far-ultraviolet (FUV, 170-300 nm) and nearultraviolet (NUV, 130-180 nm) photometric bands. A crossmatch between AGRC sample and GALEX GR6/7 provides 8899 RC stars with available observed magnitudes. Due to the lack of energy in the FUV band for many sources in the RC sample, FUV - NUV color could not be determined. Absolute magnitudes and colors are obtained for 8716 sources in NUV bands. RC stars are almost uniformly distributed in the $[\alpha / \mathrm{Fe}] \times[\mathrm{Fe} / \mathrm{H}]$ plane, which allows a fair evaluation of the different parts of this chemically defined Galactic disk. There are about 4200 stars in each chemical population for both distance subsamples $(1 / \varpi$ and BJ18). Absolute magnitude and color distributions of the GALEX NUV sample for $1 / \varpi$ and BJ18 methods are given in Figures A1 and A2, respectively. Red-colored histograms represent the high- $\alpha$ population, while blue-colored histograms represent the low- $\alpha$ populations. The Gaussian fit is shown with a green solid line and the median value in each histogram is represented with a turquoise dashed line. We will adopt the same notation for the other absolute magnitude and color distributions presented throughout the paper. The median distance of the low- $\alpha$ population is about $1.6 \mathrm{kpc}$, while, for the high- $\alpha$ population, it is about $2.3 \mathrm{kpc}$, in the $1 / \varpi$ method (Table 3 ). We find that stars in the sample 
Table 3

Results of Median Absolute Magnitudes and Colors of RC Stars in Multiwavelength Photometric Bands

\begin{tabular}{|c|c|c|c|c|c|c|c|c|c|c|c|c|c|c|c|c|c|c|c|c|c|c|c|}
\hline \multirow[b]{3}{*}{ Survey } & \multirow[b]{3}{*}{$\begin{array}{c}\text { Absolute } \\
\text { magnitude }\end{array}$} & \multicolumn{11}{|c|}{$1 / \varpi$} & \multicolumn{11}{|c|}{ BJ18 } \\
\hline & & \multicolumn{5}{|c|}{ low- $\alpha$} & \multicolumn{5}{|c|}{ high- $\alpha$} & \multirow[b]{2}{*}{$\begin{array}{l}\Delta M \\
\mathrm{mag}\end{array}$} & \multicolumn{5}{|c|}{ low- $\alpha$} & \multicolumn{5}{|c|}{ high- $\alpha$} & \multirow[b]{2}{*}{$\begin{array}{l}\Delta M \\
\mathrm{mag}\end{array}$} \\
\hline & & $\underset{\mathrm{M}}{\tilde{\mathrm{mag}}}$ & $\begin{array}{l} \pm \sigma \\
\mathrm{mag}\end{array}$ & $\begin{array}{c}\text { SE } \\
\mathrm{mag}\end{array}$ & $\begin{array}{c}N \\
\text { stars }\end{array}$ & $\begin{array}{l}\tilde{d} \\
\mathrm{pc}\end{array}$ & $\underset{\mathrm{M}}{\tilde{\mathrm{mag}}}$ & $\begin{array}{l} \pm \sigma \\
\mathrm{mag}\end{array}$ & $\begin{array}{c}\mathrm{SE} \\
\mathrm{mag}\end{array}$ & $\begin{array}{c}N \\
\text { stars }\end{array}$ & $\begin{array}{l}\tilde{d} \\
\mathrm{pc}\end{array}$ & & $\underset{\mathrm{M}}{\tilde{m}}$ & $\begin{array}{l} \pm \sigma \\
\mathrm{mag}\end{array}$ & $\begin{array}{c}\text { SE } \\
\text { mag }\end{array}$ & $\begin{array}{c}N \\
\text { stars }\end{array}$ & $\begin{array}{l}\tilde{d} \\
\mathrm{pc}\end{array}$ & $\begin{array}{c}\tilde{M} \\
\mathrm{mag}\end{array}$ & $\begin{array}{c} \pm \sigma \\
\mathrm{mag}\end{array}$ & $\begin{array}{c}\mathrm{SE} \\
\mathrm{mag}\end{array}$ & $\begin{array}{c}N \\
\text { stars }\end{array}$ & $\begin{array}{l}\tilde{d} \\
\mathrm{pc}\end{array}$ & \\
\hline GALEX & $M_{N U V}$ & 8.49 & 0.88 & 0.013 & 4400 & 1632 & 8.37 & 0.88 & 0.013 & 4316 & 2298 & 0.12 & 8.50 & 0.88 & 0.073 & 4231 & 1548 & 8.38 & 0.88 & 0.166 & 4137 & 2140 & 0.13 \\
\hline \multirow[t]{5}{*}{ SDSS } & $M_{u}$ & 4.70 & 0.84 & 0.015 & 2951 & 1168 & 4.02 & 0.82 & 0.024 & 1548 & 1793 & 0.68 & 4.78 & 0.85 & 0.025 & 1130 & 1164 & 4.24 & 0.80 & 0.032 & 615 & 1653 & 0.54 \\
\hline & $M_{g}$ & 1.45 & 0.39 & 0.007 & 2951 & 1168 & 1.54 & 0.51 & 0.015 & 1548 & 1793 & -0.09 & 1.53 & 0.41 & 0.012 & 1130 & 1164 & 1.62 & 0.44 & 0.018 & 615 & 1653 & -0.09 \\
\hline & $M_{r}$ & 0.48 & 0.26 & 0.005 & 2951 & 1168 & 0.52 & 0.32 & 0.009 & 1548 & 1793 & -0.04 & 0.55 & 0.25 & 0.007 & 1130 & 1164 & 0.64 & 0.24 & 0.010 & 615 & 1653 & -0.09 \\
\hline & $M_{i}$ & 0.20 & 0.24 & 0.004 & 2951 & 1168 & 0.25 & 0.30 & 0.009 & 1548 & 1793 & -0.05 & 0.28 & 0.24 & 0.007 & 1130 & 1164 & 0.37 & 0.22 & 0.009 & 615 & 1653 & -0.09 \\
\hline & $M_{z}$ & 0.58 & 0.34 & 0.006 & 2951 & 1168 & 0.68 & 0.43 & 0.010 & 1548 & 1793 & -0.10 & 0.65 & 0.37 & 0.011 & 1130 & 1164 & 0.79 & 0.39 & 0.016 & 615 & 1653 & -0.14 \\
\hline Gaia & $M_{G}$ & 0.45 & 0.18 & 0.002 & 11290 & 1783 & 0.51 & 0.15 & 0.004 & 6896 & 2391 & -0.06 & 0.54 & 0.21 & 0.002 & 13432 & 1738 & 0.64 & 0.19 & 0.002 & 10114 & 2243 & -0.10 \\
\hline \multirow[t]{3}{*}{ 2MASS } & $M_{J}$ & -1.17 & 0.24 & 0.002 & 12550 & 1845 & -1.05 & 0.30 & 0.003 & 9624 & 2405 & -0.12 & -1.05 & 0.21 & 0.002 & 12368 & 1751 & -0.89 & 0.27 & 0.003 & 9506 & 2244 & -0.16 \\
\hline & $M_{H}$ & -1.68 & 0.23 & 0.002 & 12550 & 1845 & -1.56 & 0.30 & 0.003 & 9624 & 2405 & -0.12 & -1.56 & 0.21 & 0.002 & 12368 & 1751 & -1.40 & 0.27 & 0.003 & 9506 & 2244 & -0.16 \\
\hline & $M_{K_{s}}$ & -1.79 & 0.22 & 0.002 & 12550 & 1845 & -1.65 & 0.30 & 0.003 & 9624 & 2405 & -0.14 & -1.67 & 0.20 & 0.002 & 12368 & 1751 & -1.50 & 0.28 & 0.003 & 9506 & 2244 & -0.17 \\
\hline \multirow[t]{4}{*}{ WISE } & $M_{W 1}$ & -1.84 & 0.22 & 0.002 & 13327 & 1632 & -1.71 & 0.30 & 0.003 & 10146 & 2298 & -0.13 & -1.72 & 0.19 & 0.002 & 12256 & 1731 & 1.55 & 0.28 & 0.003 & 9141 & 2200 & -0.17 \\
\hline & $M_{W 2}$ & -1.74 & 0.22 & 0.002 & 13438 & 1632 & -1.61 & 0.29 & 0.003 & 10228 & 2298 & -0.13 & -1.62 & 0.19 & 0.002 & 12325 & 1731 & -1.45 & 0.27 & 0.003 & 9214 & 2200 & 0.17 \\
\hline & $M_{W 3}$ & -1.85 & 0.22 & 0.002 & 12840 & 1826 & -1.74 & 0.30 & 0.003 & 8570 & 2405 & -0.11 & -1.74 & 0.20 & 0.002 & 11696 & 1682 & -1.59 & 0.28 & 0.003 & 7903 & 2066 & 0.15 \\
\hline & & & & low- $\alpha$ & & & & & high- $\alpha$ & & & & & & low- $\alpha$ & & & & & high- $\alpha$ & & & \\
\hline Survey & $\begin{array}{l}\text { Color } \\
\text { index }\end{array}$ & $\begin{array}{c}\tilde{C} \\
\mathrm{mag}\end{array}$ & $\begin{array}{c} \pm \sigma \\
\mathrm{mag}\end{array}$ & $\begin{array}{c}\mathrm{SE} \\
\mathrm{mag}\end{array}$ & $\begin{array}{c}N \\
\text { stars }\end{array}$ & & $\begin{array}{c}\tilde{C} \\
\mathrm{mag}\end{array}$ & $\begin{array}{c} \pm \sigma \\
\text { mag }\end{array}$ & $\begin{array}{c}\mathrm{SE} \\
\mathrm{mag}\end{array}$ & $\begin{array}{c}N \\
\text { stars }\end{array}$ & & $\begin{array}{l}\Delta C \\
\text { mag }\end{array}$ & $\underset{\mathrm{C}}{\tilde{\mathrm{mag}}}$ & $\begin{array}{c} \pm \sigma \\
\text { mag }\end{array}$ & $\begin{array}{c}\mathrm{SE} \\
\mathrm{mag}\end{array}$ & $\begin{array}{c}N \\
\text { stars }\end{array}$ & & $\begin{array}{c}\tilde{C} \\
\mathrm{mag}\end{array}$ & $\begin{array}{c} \pm \sigma \\
\mathrm{mag}\end{array}$ & $\begin{array}{c}\mathrm{SE} \\
\mathrm{mag}\end{array}$ & $\begin{array}{c}N \\
\text { stars }\end{array}$ & & $\begin{array}{l}\Delta C \\
\mathrm{mag}\end{array}$ \\
\hline GALEX & $\ldots$ & $\ldots$ & $\ldots$ & $\cdots$ & $\cdots$ & $\ldots$ & $\ldots$ & $\cdots$ & $\ldots$ & $\ldots$ & $\cdots$ & $\ldots$ & $\ldots$ & $\cdots$ & $\ldots$ & $\ldots$ & $\cdots$ & $\ldots$ & $\ldots$ & $\ldots$ & $\cdots$ & $\ldots$ & $\cdots$ \\
\hline \multirow[t]{4}{*}{ SDSS } & $(u-g)_{o}$ & 3.32 & 0.83 & 0.015 & 2951 & & 2.57 & 0.98 & 0.029 & 1548 & & 0.75 & 3.37 & 0.88 & 0.026 & 1130 & & 2.68 & 0.89 & 0.036 & 615 & & 0.69 \\
\hline & $(g-r)_{o}$ & 0.92 & 0.18 & 0.003 & 2951 & & 0.87 & 0.16 & 0.005 & 1548 & & 0.05 & 0.93 & 0.18 & 0.005 & 1130 & & 0.87 & 0.16 & 0.006 & 615 & & 0.06 \\
\hline & $(r-i)_{o}$ & 0.28 & 0.03 & 0.001 & 2951 & & 0.28 & 0.03 & 0.001 & 1548 & & 0.00 & 0.28 & 0.03 & 0.001 & 1130 & & 0.27 & 0.03 & 0.001 & 615 & & 0.01 \\
\hline & $(i-z)_{o}$ & -0.34 & 0.25 & 0.005 & 2951 & & -0.37 & 0.28 & 0.008 & 1548 & & 0.03 & -0.32 & 0.24 & 0.007 & 1130 & & -0.35 & 0.27 & 0.011 & 615 & & 0.03 \\
\hline Gaia & $\left(G_{B P}-G_{R P}\right)_{o}$ & 1.22 & 0.04 & 0.001 & 11290 & & 1.21 & 0.04 & 0.001 & 6896 & & 0.01 & 1.22 & 0.04 & 0.001 & 13432 & & 1.20 & 0.06 & 0.001 & 10114 & & 0.02 \\
\hline \multirow[t]{3}{*}{ 2MASS } & $\left(J-K_{s}\right)_{o}$ & 0.62 & 0.05 & 0.001 & 12550 & & 0.62 & 0.05 & 0.001 & 9624 & & 0.00 & 0.62 & 0.05 & 0.001 & 12368 & & 0.62 & 0.05 & 0.001 & 9506 & & 0.00 \\
\hline & $\left(H-K_{s}\right)_{o}$ & 0.10 & 0.03 & 0.001 & 12550 & & 0.10 & 0.03 & 0.001 & 9624 & & 0.00 & 0.10 & 0.03 & 0.001 & 12368 & & 0.10 & 0.03 & 0.001 & 9506 & & 0.00 \\
\hline & $(J-H)_{o}$ & 0.51 & 0.04 & 0.001 & 12550 & & 0.52 & 0.04 & 0.001 & 9624 & & -0.01 & 0.51 & 0.04 & 0.001 & 12368 & & 0.52 & 0.04 & 0.001 & 9506 & & -0.01 \\
\hline \multirow[t]{3}{*}{ WISE } & $(W 1-W 2)_{o}$ & -0.10 & 0.02 & 0.001 & 13327 & & -0.09 & 0.02 & 0.001 & 10146 & & -0.01 & -0.10 & 0.02 & 0.001 & 13309 & & -0.09 & 0.02 & 0.001 & 10126 & & -0.01 \\
\hline & $(W 1-W 3)_{o}$ & 0.01 & 0.04 & 0.001 & 12539 & & 0.03 & 0.04 & 0.001 & 8478 & & -0.02 & 0.01 & 0.04 & 0.001 & 12526 & & 0.03 & 0.04 & 0.001 & 8469 & & -0.02 \\
\hline & $(W 2-W 3)_{o}$ & 0.11 & 0.03 & 0.001 & 12643 & & 0.12 & 0.04 & 0.001 & 8552 & & -0.01 & 0.11 & 0.03 & 0.001 & 12629 & & 0.12 & 0.04 & 0.001 & 8543 & & -0.01 \\
\hline
\end{tabular}

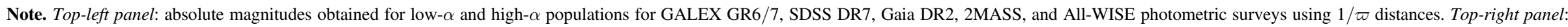

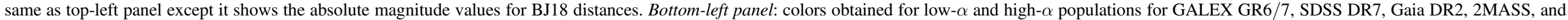

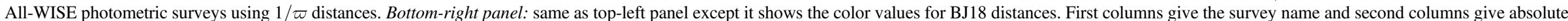

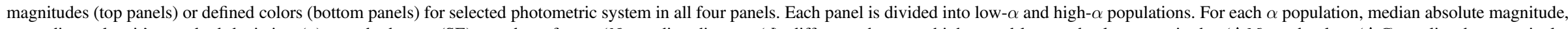

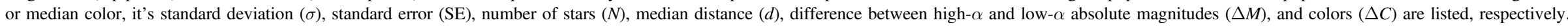


Table 4

Literature Comparison of Absolute Magnitudes in Near-UV and Optical Bands Obtained from Low- $\alpha$ and High- $\alpha$ Populations for 1/ $\varpi$ and BJ18 Methods

\begin{tabular}{|c|c|c|c|c|c|c|c|}
\hline GALEX & $N$ (stars) & $M_{N U V}(\mathrm{mag})$ & & & & & Remarks \\
\hline This study low- $\alpha$ & 1632 & $8.49 \pm 0.013$ & & & & & Gaia DR2 $(1 / \varpi)$ \\
\hline This study high- $\alpha$ & 2298 & $8.37 \pm 0.013$ & & & & & Gaia DR2 $(1 / \varpi)$ \\
\hline This study low- $\alpha$ & 1548 & $8.50 \pm 0.073$ & & & & & Gaia DR2 (BJ18) \\
\hline This study high- $\alpha$ & 2140 & $8.38 \pm 0.166$ & & & & & Gaia DR2 (BJ18) \\
\hline SDSS & $N$ (stars) & $M_{u}(\mathrm{mag})$ & $M_{g}(\mathrm{mag})$ & $M_{r}(\mathrm{mag})$ & $M_{i}(\mathrm{mag})$ & $M_{z}(\mathrm{mag})$ & Remarks \\
\hline Chen et al. (2017) & 171 & $\ldots$ & $1.229 \pm 0.172$ & $0.420 \pm 0.110$ & $0.157 \pm 0.094$ & $0.022 \pm 0.084$ & 171 APASS ugriz \\
\hline Ruiz-Dern et al. (2018) & 1315 & $\cdots$ & $1.331 \pm 0.056$ & $0.552 \pm 0.026$ & $0.262 \pm 0.032$ & $\cdots$ & TGAS Gaia DR1 \\
\hline This study low- $\alpha$ & 1168 & $4.70 \pm 0.015$ & $1.45 \pm 0.007$ & $0.48 \pm 0.005$ & $0.20 \pm 0.004$ & $0.58 \pm 0.006$ & Gaia DR2 $(1 / \varpi)$ \\
\hline This study high- $\alpha$ & 1793 & $4.02 \pm 0.024$ & $1.54 \pm 0.015$ & $0.52 \pm 0.009$ & $0.25 \pm 0.009$ & $0.68 \pm 0.010$ & Gaia DR2 $(1 / \varpi)$ \\
\hline This study low- $\alpha$ & 1164 & $4.78 \pm 0.025$ & $1.53 \pm 0.012$ & $0.55 \pm 0.007$ & $0.28 \pm 0.007$ & $0.65 \pm 0.011$ & Gaia DR2 (BJ18) \\
\hline This study high- $\alpha$ & 1653 & $4.24 \pm 0.032$ & $1.62 \pm 0.018$ & $0.64 \pm 0.010$ & $0.37 \pm 0.009$ & $0.79 \pm 0.016$ & Gaia DR2 (BJ18) \\
\hline Gaia & $N$ (stars) & $M_{G}(\mathrm{mag})$ & & & & & Remarks \\
\hline Hawkins et al. (2017) & 972 & $0.44 \pm 0.01$ & & & & & TGAS \\
\hline Ruiz-Dern et al. (2018) & 2482 & $0.495 \pm 0.009$ & & & & & TGAS \\
\hline This study low- $\alpha$ & 11290 & $0.45 \pm 0.002$ & & & & & Gaia DR2 $(1 / \varpi)$ \\
\hline This study high- $\alpha$ & 6896 & $0.51 \pm 0.004$ & & & & & Gaia DR2 $(1 / \varpi)$ \\
\hline This study low- $\alpha$ & 13432 & $0.54 \pm 0.002$ & & & & & Gaia DR2 (BJ18) \\
\hline This study high- $\alpha$ & 10114 & $0.64 \pm 0.002$ & & & & & Gaia DR2 (BJ18) \\
\hline
\end{tabular}

Note. Columns give survey, number of stars, absolute magnitudes, and remarks that include the data source.

have similar absolute magnitudes in NUV for low- and high- $\alpha$ regimes in both distance subsamples. Moreover, if each distance subsample is considered separately, the effect of the variation of chemical population causes $0.12 \leqslant \Delta M \leqslant 0.13$ mag variation in both distance method.

No literature value to compare with $M_{\mathrm{NUV}}$ magnitudes of this study is found except Mohammed et al.'s (2019) study. In their study, GALEX and Gaia photometric data are combined, and the color-metallicity relation is obtained for RC stars. However their results are incomparable with our study because they give only the relation between $(N U V-G)_{0}$ and $[\mathrm{Fe} / \mathrm{H}]$. We made an inner comparison between $1 / \varpi$ and BJ18 methods for low- $\alpha$ and high- $\alpha$ magnitudes, which suggests that the UV peaks around the same median value with a very similar distribution for both chemical thin and thick disk populations.

\subsection{Optical Region}

In this section, we present the results obtained using the SDSS DR7 and Gaia DR2 photometric bands. The Sloan photometric system covers $\lambda \lambda 300-1000 \mathrm{~nm}$ wavelength range with five photometric bands, i.e., $u, g, r, i, z$. Four photometric colors are defined from these bands, $(u-g)_{o},(g-r)_{o}$, $(r-i)_{o}$, and $(i-z)_{o}$ (Fukugita et al. 1996; Fan 1999). The cross-match between the AGRC spectral catalog and the SDSS DR7 photometric catalog returns 4499 common RC stars. The distributions of SDSS absolute magnitudes are given in Figures A3 and A4 for $1 / \varpi$ and BJ18 methods, respectively. Similarly, the distributions of SDSS colors are given in Figures A5 and A6 for $1 / \varpi$ and BJ18 methods, respectively. There are almost two times more stars in the low- $\alpha$ than in the high- $\alpha$ population. For the distances estimated by the $1 / \varpi$ and BJ18 method, absolute magnitudes in the SDSS $u$ band appear distinct in behavior from the rest of the remaining bands. The lower sensitivity of the $u$ band does not allow a more reliable data evaluation. Our results indicate that the high- $\alpha$ population is brighter than the low- $\alpha$ population in the $u$ band. In the $g$ band, RC stars become brighter by 0.09 mag as the chemical population changes from low- to high- $\alpha$. A similar trend is followed by $r, i$, and $z$ bands, with an increase in brightness by $0.04,0.05$, and $0.10 \mathrm{mag}$, respectively. This relatively larger absolute magnitude difference value in the $z$ band might be caused by low response of this photometric band, which is shown in Figure 7 with an orange dashed line.

No literature value is found for the $u$ band to compare, as can be seen from Table 4 , but the inner comparison between $1 / \varpi$ and BJ18 methods for the chemically thin disk suggest that there is a $0.08 \mathrm{mag}$ difference while, for the chemically thick disk, the difference is even larger, up to $0.22 \mathrm{mag}$. SDSS $M_{g}$, $M_{r}, M_{i}$, and $M_{z}$ magnitudes are compared with the results of Chen et al. (2017) with 171 RC stars with APASS Sloan photometry, and the result of Ruiz-Dern et al. (2018) with 1315 TGAS DR1 RC stars. These studies are focused on the thin disk sample, so that their results are useful only for the low- $\alpha$ populations. $M_{g}$ magnitudes of Chen et al. (2017) and RuizDern et al. (2018) are brighter by about 0.20 mag than our low$\alpha \mathrm{RCs}$ for the $1 / \varpi$ method. These values are even larger (by $0.08 \mathrm{mag}$ ) when we consider the results obtained with BJ18 method. Absolute magnitudes in $r$ and $i$ bands coincide with the chemical thin disk values obtained with BJ18's method. On the other hand, the absolute magnitudes in $z$ band are quite different $\left(\Delta M_{z}>0.60 \mathrm{mag}\right)$ from the Chen et al. (2017) result.

Absolute magnitudes and colors in optical bands are further analyzed using the Gaia $G$ band magnitude and $\left(G_{B P}-G_{R P}\right)_{o}$ color, which covers the same wavelength range of SDSS, but with much broader photometric bands. After the chemical populations are separated, $62 \%$ of Gaia sample stars are in the low- $\alpha$ population while $38 \%$ is in the high- $\alpha$ population, which is expected due to the astrometric precision of this RC sample. The distributions for the $G$ band absolute magnitude and $\left(G_{B P}-G_{R P}\right)_{o}$ de-reddened color are shown in Figures $\mathrm{A} 7$ and A8, respectively. The $G$ band absolute magnitudes increase by $0.06 \mathrm{mag}$ for the $1 / \varpi$ method while this difference is $0.1 \mathrm{mag}$ for the BJ18 method, from low- $\alpha$ to high- $\alpha$ population 

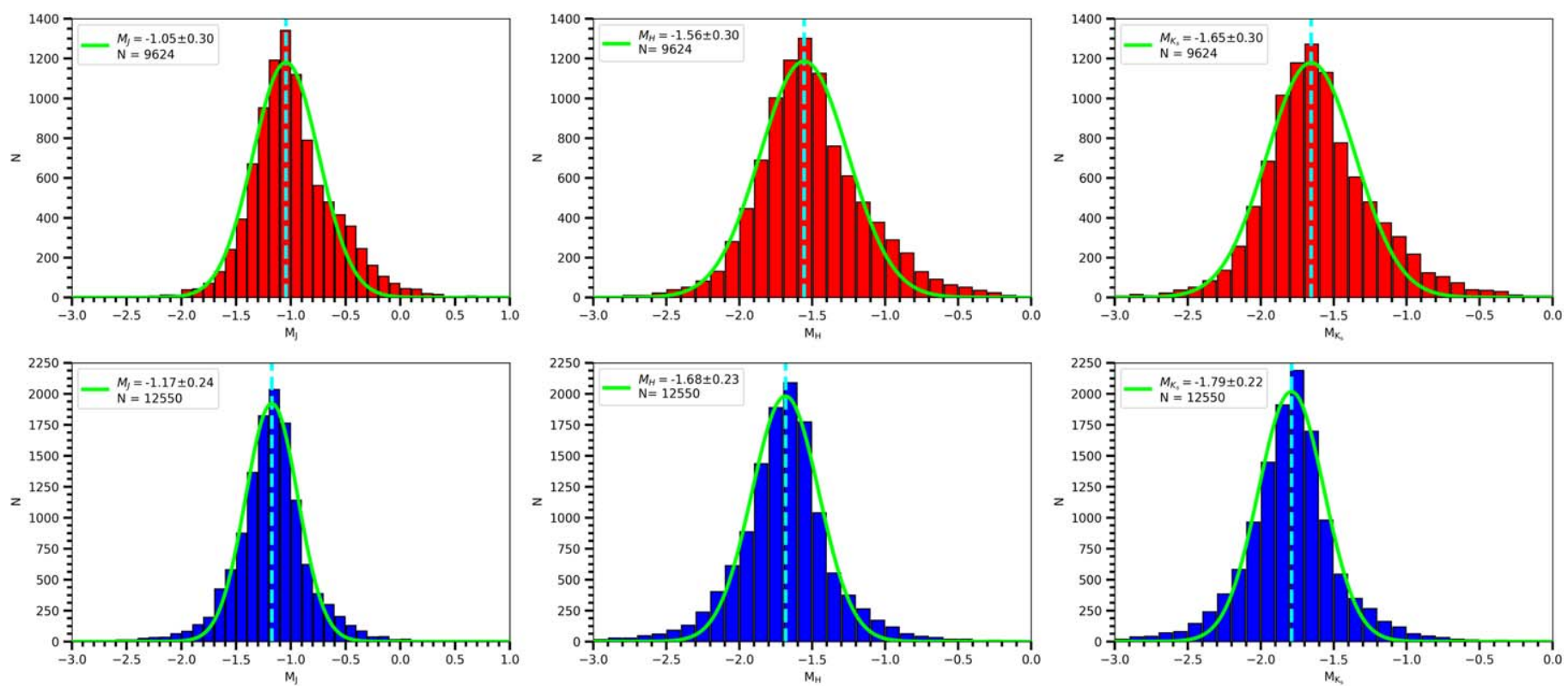

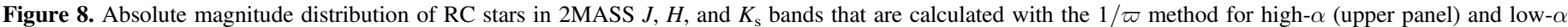
(lower panel) populations. Green solid line is the Gaussian fit for the distribution and turquoise dashed line is the median value of the distribution.

(Table 3). On the other hand, $\left(G_{B P}-G_{R P}\right)_{o}$ colors appear to be not affected by either the chemical population or the distance estimation method. As seen Table 3, the resulting color difference is almost constant for both methods.

In the Gaia $G$ band, the absolute magnitude for low- $\alpha$ RC stars for the $1 / \varpi$ method seems to be in good agreement with the Hawkins et al. (2017) and Ruiz-Dern et al. (2018)'s results that were obtained using TGAS catalog (Michalik et al. 2015). Optic and UV absolute magnitudes and literature comparison is given in Table 4.

\subsection{Infrared Region}

For the analysis of the NIR region, we use the 2MASS photometric bands $J(1.25 \mu \mathrm{m}), H(1.65 \mu \mathrm{m})$, and $K_{s}(2.17 \mu \mathrm{m})$. Stars with photometric quality AAA are selected for their more accurate photometry (Cutri et al. 2003). More than 22,000 RC stars are cross-matched with AGRC spectral catalog and they are almost equally distributed in the chemical space. Three absolute magnitudes, i.e., $M_{J}, M_{H}$, and $M_{K_{s}}$ and three dereddened color indices, i.e., $\left(J-K_{s}\right)_{o},(J-H)_{o}$ and $\left(H-K_{S}\right)_{o}$ are calculated with the method described in Section 4. For 2MASS photometry, most of the RC sample have all three apparent magnitudes. Thus there is a unique number of stars for each chemical population under each distance estimation method. The low- $\alpha$ population contains more than 12,500 RC stars while high- $\alpha$ population has about 9600 RC stars. Based on the absolute magnitude frequency distributions, which are given in Figures 8 and 9, chemically thin disk RC stars are brighter by 0.12 or 0.14 mag than the chemically thick disk stars once the distances are estimated via $1 / \varpi$ method. On the other hand, this value is pushed to 0.16 or 0.17 mag difference when using the BJ18 method. Based on the color distributions given in Figures A9 and A10, there is no clear variation in 2MASS colors (Table 3).

Literature values are compiled in Table 5 for NIR and MIR regions. The standard candle status of RC stars is extensively studied with 2MASS photometry since the beginning of the new millennium. Early studies used the astrometric data provided by Hipparcos survey, TGAS, or open cluster data. According to the literature studies, $M_{J}$ values vary between -0.93 to -1.02 mag for the thin disk (Laney et al. 2012; Bilir et al. 2013a; Karaali et al. 2013; Yaz Gökçe et al. 2013; Chen et al. 2017; Hawkins et al. 2017; Ruiz-Dern et al. 2018). The median $M_{J}$ value for the chemically thin disk is -1.17 mag (for $1 / \varpi)$, which is $0.15 \mathrm{mag}$ brighter than that in the literature while, for the BJ18 method, it is $-1.05 \mathrm{mag}$, which is closer to the literature values. Absolute magnitudes in the $H$-band vary between -1.45 and -1.53 mag (see Table 5). The $M_{H}$ absolute magnitudes of the low- $\alpha$ subsample (for $1 / \varpi$ ) are cover 0.15 and 0.23 mag brighter than the existing values in literature, respectively. On the other hand, the low- $\alpha$ subsample (for BJ18) is close to the values of Chen et al. (2017)'s RC stars that were selected with asteroseismology methods. $M_{K_{s}}$ is the most studied magnitude to test the standard candle status of RC stars. The majority of literature studies agree on $M_{K_{s}}=-1.61 \mathrm{mag}$ value (Alves 2000; Grocholski \& Sarajedini 2002; Laney et al. 2012; Yaz Gökçe et al. 2013; Chen et al. 2017; Hawkins et al. 2017; Ruiz-Dern et al. 2018) while other studies give brighter values but still within the error bars. However, the results for $M_{K_{s}}$ absolute magnitude obtained in this study are -1.79 and $-1.67 \mathrm{mag}$ for the chemically thin disk and -1.65 and -1.50 mag for the chemically thick disk. These values are not in agreement with any literature values.

For the analysis of the MIR region, we use the four WISE photometric bands, $W 1 \quad(3.368 \mu \mathrm{m}), W 2(4.618 \mu \mathrm{m}), W 3$ $(12.082 \mu \mathrm{m})$, and $W 4(22.194 \mu \mathrm{m})$ and three colors, i.e., $(W 1-W 2)_{0},(W 1-W 3)_{0}$, and $(W 2-W 3)_{0}$. The WISE sample is selected to obtain an optimum number of stars in each photometric band. Overall, there are more objects in the low- $\alpha$ population $(\approx 12,500)$ than high- $\alpha(\approx 9200)$ for both distance method in WISE photometry. However, for W4 photometric band, the number of RC stars are not sufficient to perform a statistically meaningful analysis. Frequency distribution of absolute magnitudes in $W 1, W 2$, and $W 3$ photometric bands are shown in Figures A11 and A12. 

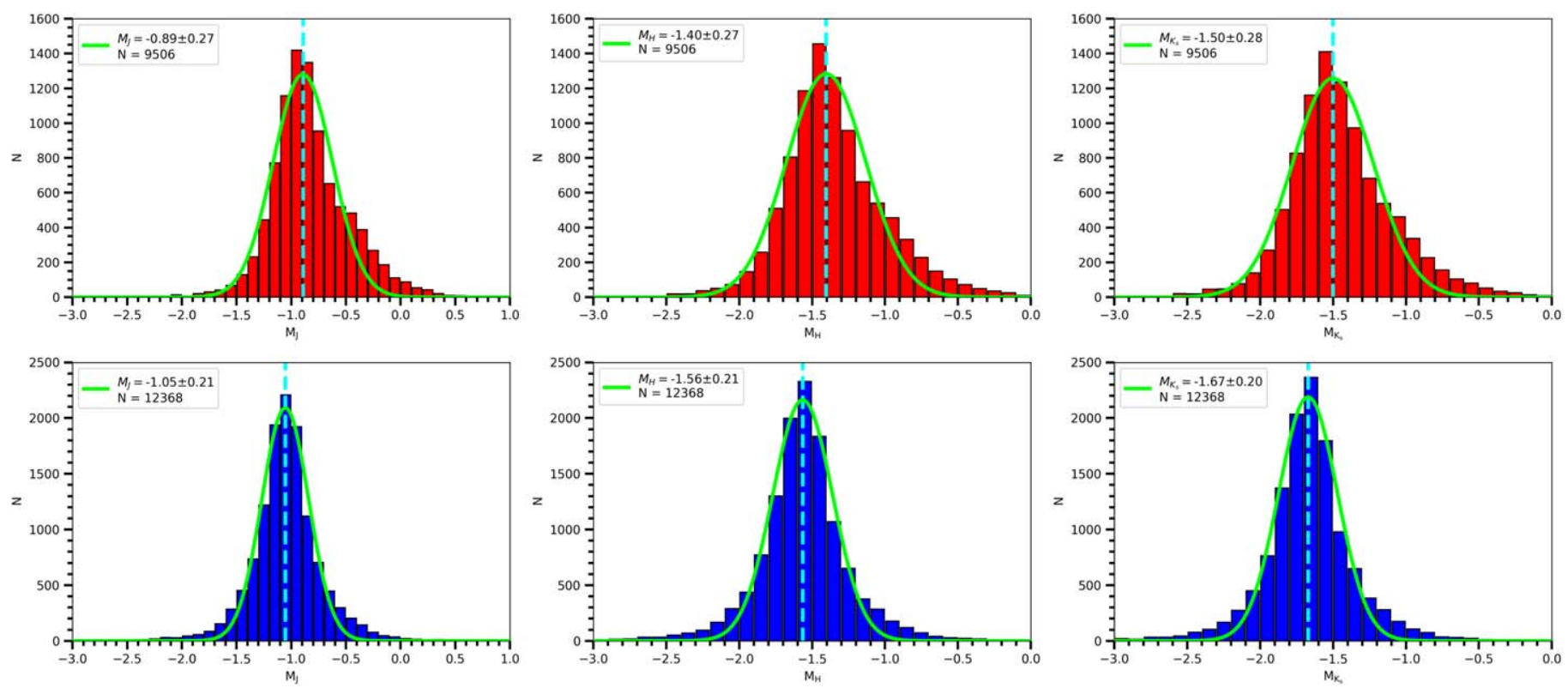

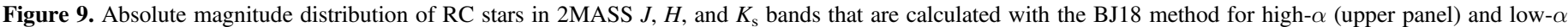
(lower panel) populations. Green solid line is the Gaussian fit for the distribution and turquoise dashed line is the median value of the distribution.

Absolute magnitudes vary -0.11 and -0.13 mag for WISE bands as the population changes from low- to high- $\alpha$ for $1 / \varpi$ method. This difference increases between -0.15 and -0.17 mag using the BJ18 method. Regardless of the number of RC stars, colors are changed mildly $(<0.02 \mathrm{mag})$ from low- to high- $\alpha$ population, as shown in Figures A13 and A14.

WISE absolute magnitudes are obtained from the samples with Hipparcos trigonometric parallaxes and with relatively small RC samples (less than 4000), Three of the four studies obtain the distances using photometric parallax, inverse parallax, and InfraRed flux methods. Based on the literature results in Table 5, for the $M_{W 1}$ values by Chen et al. (2017), Hawkins et al. (2017), and Ruiz-Dern et al. (2018), results scatter around $-1.69 \mathrm{mag}$, while Yaz Gökçe et al.'s (2013) $M_{W 1}$ is $-1.61 \mathrm{mag}$. For the BJ18 method, the value for the chemical thin disk sample is in agreement literature, but it is 0.10 mag brighter for the chemically thin disk of the $1 / \varpi$ method. In $M_{W 2}$, the chemically thin disk of BJ18 is in agreement with Chen et al. (2017) and Ruiz-Dern et al. (2018), while the chemically thin disk of $1 / \varpi$ is in accordance with Hawkins et al.'s (2017) studies.

Overall comparison shows that, regardless of the distance method, we found a general trend of increasing luminosity from shorter to longer wavelengths, with a relative magnitude variation between chemical population.

\section{Testing Absolute Magnitudes with a Mock Catalog}

The mock data is generated with the Besançon population synthesis model (Robin et al. 2003, 2012). This model provides theoretical density distribution for stars in the Galactic bulge, bar, thin disk, thick disk, and halo. The most up-to-date version of the model is run for the most crowded Galactic region in our AGRC catalog, $90^{\circ} \leqslant l \leqslant 270^{\circ}$. This model is chosen because it can produce $[\alpha / \mathrm{Fe}]$ with stellar atmospheric parameters. These parameters allow us to apply the same procedure to select synthetic RC stars that belong to different chemical populations from the mock sample, such as our AGRC catalog.
With its high signal-to-noise ratio, 2MASS photometry is chosen as a base photometric system for APOGEE and GALAH spectroscopic surveys to compare the model with observational data. Model results are plotted on the HR diagram and RC stars are selected with the same method used in Section 2.1. Chemical population separation is done by applying the same criteria used in Section 3 to the mock catalog. In Figure 10, absolute magnitudes for 2MASS photometry of the mock catalog are shown for low- and high- $\alpha$ RC populations. Results of this mock sample indicate that the observed absolute magnitude differences in both populations still hold like in the observational data. However, the difference is more amplified, i.e., $\langle\Delta M\rangle=-0.37$ mag. Comparison between the observational and theoretical absolute magnitudes in 2MASS bands is given in Table 6.

\section{RC Contamination}

RC stars are metal-rich horizontal branch stars, that span a narrow range on $\log g$ and a relatively wide range on $T_{\text {eff. In }}$ this study, the most probable RC stars are selected using multiple Gaussian distributions on $T_{\text {eff }}$ and $\log g$ parameters. $\mathrm{RC}$ selection is done by selecting stars within the $2 \sigma$ region around the central coordinates of these distributions. In Figure 2, there is an evident peak of $\mathrm{RC}$ on the $\log g$ distribution and there is a large overlapping region with RGB stars below this distribution. In contrast, the RC population appears reasonably well separated in the distribution on the $T_{\text {eff }}$. Based on these distributions, the RC sample seems to be contaminated with a small percentage of sub-giants and RGB stars, but these subsamples reside in the $3 \sigma$ region of the central coordinates of the $\log g \times T_{\text {eff }}$ distribution.

Contamination of the AGRC sample is also tested using the empirical method of Holtzman et al. (2018), the method that considers the $[\mathrm{C} / \mathrm{N}]$ ratio as a proxy for mass (Ness et al. 2015; Martig et al. 2016) to identify RGB stars. In this method, a reference temperature is calculated with their Equation (10). Then $T_{\text {eff }}$ and $\log g$ are linked to the $[\mathrm{C} / \mathrm{N}]$ abundance ratio in order to assign the stellar population using Equation (11) of 

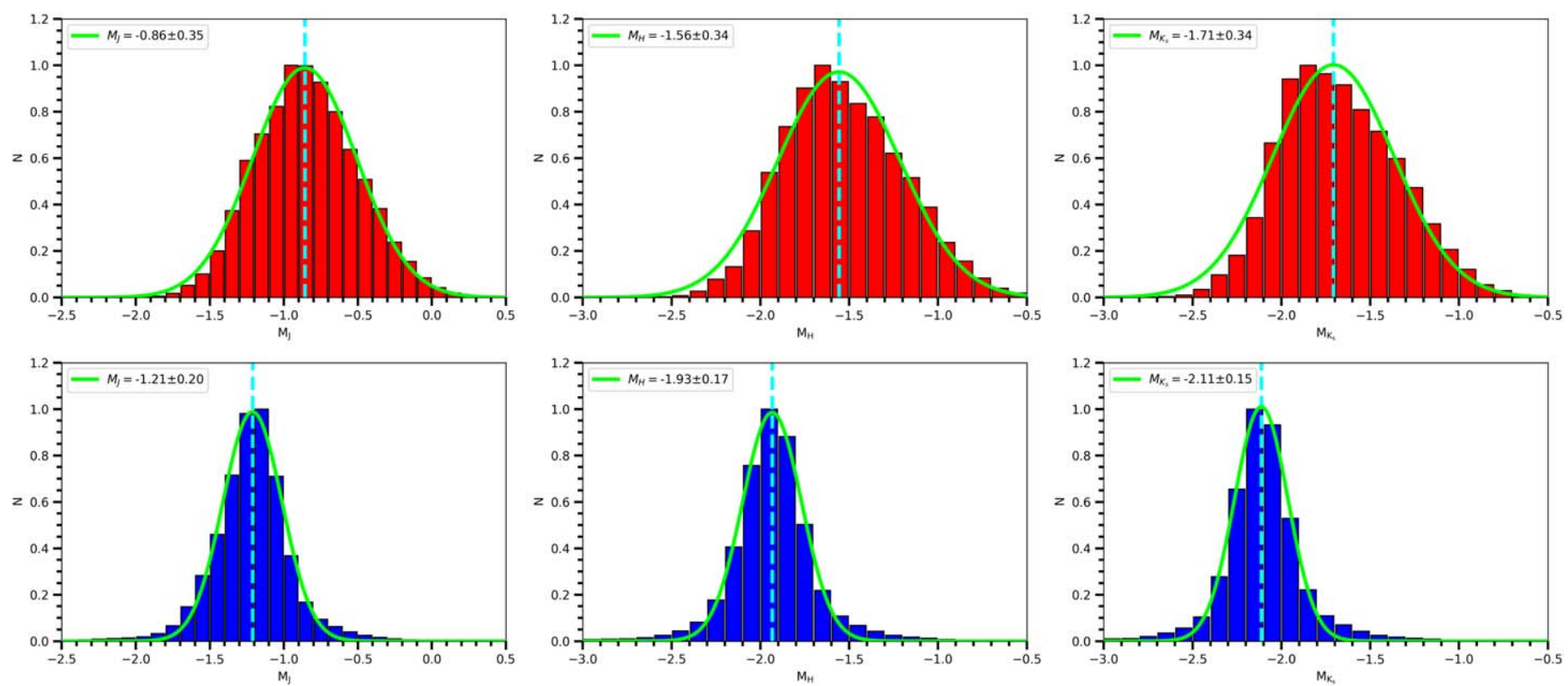

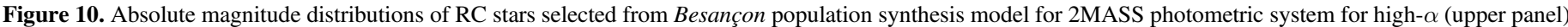
and low- $\alpha$ (lower panel) populations.

Table 5

Literature Comparison of Absolute Magnitudes in NIR and MIR Bands Obtained from Low- $\alpha$ and High- $\alpha$ Populations for $1 / \varpi$ and BJ18 Methods

\begin{tabular}{|c|c|c|c|c|c|c|}
\hline 2MASS & $N$ (stars) & $M_{J}(\mathrm{mag})$ & $M_{H}(\mathrm{mag})$ & $M_{K_{s}}(\mathrm{mag})$ & & Remarks \\
\hline Alves (2000) & 238 & $\cdots$ & $\ldots$ & $-1.61 \pm 0.03$ & & Hipparcos \\
\hline Grocholski \& Sarajedini (2002) & 14 & $\cdots$ & $\cdots$ & $-1.61 \pm 0.04$ & & $\begin{array}{l}\text { WYIN open } \\
\text { clusters }\end{array}$ \\
\hline $\begin{array}{l}\text { van Helshoecht \& Groenewegen } \\
\text { (2007) }\end{array}$ & 24 & $\cdots$ & $\cdots$ & $-1.57 \pm 0.05$ & & $\begin{array}{l}\text { 2MASS open } \\
\text { clusters }\end{array}$ \\
\hline Cabrera-Lavers et al. (2007b) & $49^{*}$ & $\ldots$ & $\ldots$ & $-1.62 \pm 0.03$ & & TCS-CAIN \\
\hline Groenewegen (2008) & & $\cdots$ & $\cdots$ & $-1.54 \pm 0.04$ & & revised Hipparcos \\
\hline Laney et al. (2012) & 191 & $-0.984 \pm 0.014$ & $-1.490 \pm 0.015$ & $-1.613 \pm 0.015$ & & revised Hipparcos \\
\hline Yaz Gökçe et al. (2013) & 2937 & $-0.970 \pm 0.016$ & $-1.462 \pm 0.014$ & $-1.595 \pm 0.025$ & & revised Hipparcos \\
\hline Francis \& Anderson (2014) & & $\cdots$ & $\cdots$ & $-1.53 \pm 0.01$ & & revised Hipparcos \\
\hline Chen et al. (2017) & 171 & $-1.016 \pm 0.063$ & $-1.528 \pm 0.055$ & $-1.626 \pm 0.057$ & & SAGA \\
\hline Hawkins et al. (2017) & 972 & $-0.93 \pm 0.01$ & $-1.46 \pm 0.01$ & $-1.61 \pm 0.01$ & & TGAS \\
\hline Ruiz-Dern et al. (2018) & 2482 & $-0.945 \pm 0.01$ & $-1.450 \pm 0.017$ & $-1.606 \pm 0.009$ & & TGAS \\
\hline This study low- $\alpha$ & 12550 & $-1.17 \pm 0.002$ & $-1.68 \pm 0.002$ & $-1.79 \pm 0.002$ & & Gaia DR2 $(1 / \varpi)$ \\
\hline This study high- $\alpha$ & 9624 & $-1.05 \pm 0.003$ & $-1.56 \pm 0.003$ & $-1.65 \pm 0.003$ & & Gaia DR2 $(1 / \varpi)$ \\
\hline This study low- $\alpha$ & 12638 & $-1.05 \pm 0.002$ & $-1.56 \pm 0.002$ & $-1.67 \pm 0.002$ & & Gaia DR2 (BJ18) \\
\hline This study high- $\alpha$ & 9506 & $-0.89 \pm 0.003$ & $-1.40 \pm 0.003$ & $-1.50 \pm 0.003$ & & Gaia DR2 (BJ18) \\
\hline WISE & $N$ (stars) & $M_{W 1}(\mathrm{mag})$ & $M_{W 2}(\mathrm{mag})$ & $M_{W 3}(\mathrm{mag})$ & $M_{W 4}(\mathrm{mag})$ & Remarks \\
\hline Yaz Gökçe et al. (2013) & 3889 & $-1.612 \pm 0.022$ & $\cdots$ & $-1.585 \pm 0.019$ & $\cdots$ & revised Hipparcos \\
\hline Chen et al. (2017) & $<171$ & $-1.694 \pm 0.061$ & $-1.595 \pm 0.064$ & $-1.752 \pm 0.068$ & $\ldots$ & SAGA \\
\hline Hawkins et al. (2017) & $>900$ & $-1.68 \pm 0.02$ & $-1.69 \pm 0.02$ & $-1.67 \pm 0.01$ & $-1.76 \pm 0.01$ & TGAS \\
\hline Ruiz-Dern et al. (2018) & $\begin{array}{l}962,1032 \\
2026,747\end{array}$ & $-1.711 \pm 0.017$ & $-1.585 \pm 0.016$ & $-1.638 \pm 0.011$ & $-1.704 \pm 0.012$ & TGAS \\
\hline This study low- $\alpha$ & {$[12840,13438]$} & $-1.84 \pm 0.002$ & $-1.74 \pm 0.002$ & $-1.85 \pm 0.002$ & $\cdots$ & Gaia DR2 $(1 / \varpi)$ \\
\hline This study high- $\alpha$ & {$[8570,10228]$} & $-1.71 \pm 0.003$ & $-1.61 \pm 0.003$ & $-1.74 \pm 0.003$ & $\cdots$ & Gaia DR2 $(1 / \varpi)$ \\
\hline This study low- $\alpha$ & {$[11696,12325]$} & $-1.72 \pm 0.002$ & $-1.62 \pm 0.002$ & $-1.74 \pm 0.002$ & $\cdots$ & Gaia DR2 (BJ18) \\
\hline This study high- $\alpha$ & {$[7903,9214]$} & $-1.55 \pm 0.003$ & $-1.45 \pm 0.003$ & $-1.59 \pm 0.003$ & $\cdots$ & Gaia DR2 (BJ18) \\
\hline
\end{tabular}

Note. Columns give survey, number of stars, absolute magnitudes, and remarks that include the data source. ${ }^{*}$ represents star fields.

Holtzman et al. (2018). In this study, this method is only applied to the APOGEE RC stars, because the APOGEE survey provides both carbon and nitrogen abundances while the GALAH survey provides only carbon. So, this separation method is only applied to the APOGEE giants. Based on the analysis of 10,760 RC giants, the contamination of RGB stars is found to be $0.35 \%$. Instead, the contamination levels of GALAH RC stars are unknown.

In recent years Bovy et al. (2014) provided a new catalog of RC stars compiled from APOGEE DR11 by applying a new 
Table 6

Comparison between 2MASS $M_{J}, M_{H}$, and $M_{K_{S}}$ Absolute Magnitudes Generated with Besançon Population Synthesis Model and AGRC Catalog for Low- $\alpha$ and High- $\alpha$ Populations

\begin{tabular}{|c|c|c|c|c|c|c|}
\hline & \multicolumn{2}{|c|}{ Gaia } & \multicolumn{2}{|c|}{ Besançon } & \multicolumn{2}{|c|}{$\Delta M(\mathrm{mag})$} \\
\hline & low- $\alpha$ & high- $\alpha$ & low- $\alpha$ & high- $\alpha$ & observed & model \\
\hline$\overline{M_{J}}$ & -1.17 & -1.05 & -1.21 & -0.86 & -0.12 & -0.35 \\
\hline$M_{H}$ & -1.68 & -1.56 & -1.93 & -1.56 & -0.12 & -0.37 \\
\hline$M_{K_{s}}$ & -1.79 & -1.65 & -2.11 & -1.71 & -0.14 & -0.40 \\
\hline$N$ & \multicolumn{2}{|c|}{45,877} & \multicolumn{2}{|c|}{342,502} & & \\
\hline
\end{tabular}

Note. Absolute magnitude errors are less than 0.003 mag.

technique on spectrophotometric data. This catalog is further updated in APOGEE DR15 and has 5\% contamination.

Our AGRC sample coincides with the $65 \%$ of Bovy's RC catalog of APOGEE DR15. The remaining 35\% fraction is missing because of (i) application of relative parallax error limitation to our sample $\left(\sigma_{\varpi} / \varpi \leqslant 0.1\right.$ and (ii) differences between stellar atmospheric model parameters arising because the original RC catalog of Bovy et al. (2014), which uses APOGEE DR11, is extended to the DR15 data release. The average difference in $T_{\text {eff }}$ is $55 \mathrm{~K}$ with $\sigma_{T_{\text {eff }}}=45 \mathrm{~K}$ while the average difference in logarithmic surface gravity is $0.1 \mathrm{dex}$ with $\sigma_{\log g}=0.15$ dex. These differences can explain why only $65 \%$ of the stars matched.

\section{Summary and Conclusion}

In this study, absolute magnitudes and colors of RC stars selected from high-resolution and high S/N APOGEE DR14 (Majewski et al. 2017) and GALAH DR2 (de Silva et al. 2016) spectroscopic surveys, are examined in a range of UV, optical, NIR, and MIR photometric bands of GALEX GR6/7 (Bianchi et al. 2017), SDSS DR7 (Abazajian et al. 2009), Gaia DR2 (Gaia Collaboration et al. 2018), 2MASS (Cutri et al. 2003), and All-WISE (Cutri et al. 2013) photometric surveys, respectively. In this study, RC stars are not used as standard candles. Instead, their standard candle value is thoroughly examined. The RC star distances are individually estimated with $1 / \varpi$ and BJ18 method by using Gaia DR2 parallaxes.

This study provides a new method to select RC stars using overlapping Gaussian distributions on the spectroscopic plane. Also, the separation of RC stars into the chemical populations is done by running a machine-learning algorithm that produces a decision boundary line to provide a clear separation on the chemical plane. RC stars are identified using the Gaussian distributions of each contributing parameter of the HR diagram. Initially, sources with $\sigma_{\varpi} / \varpi \leqslant 0.1$ are selected to obtain a more accurate astrometric sample. This procedure is followed by the application of the GMM method to separate RC stars into low- $\alpha$ (thin disk) stars and high- $\alpha$ (thick disk) stars. By doing so, chemical space solves most of the Galactic population separation problem, which is the best choice so far; this approach has never been used before in the literature. The RC sample is further cross-matched with GALEX GR6/7, SDSS DR7, Gaia DR2, 2MASS, and All-WISE catalogs and all of the subsamples are evaluated in their own unique conditions, which, in turn, is given the non-unique number of sources in each photometric survey.
Results are evaluated for each photometric band and color (i) by comparing absolute magnitude values in low- and high- $\alpha$ populations, (ii) by comparing different distance estimation methods, and (iii) by comparing observational results for 2MASS $J, H$, and $K_{s}$ bands with a mock catalog generated for the current known parameters of our Galaxy.

The overall comparison of the absolute magnitudes and colors with the systematic analysis that is used in this study indicates that there exist specific trends between low- and high$\alpha$ populations, regardless of the distance estimation method. A general trend of brightening in the UV region of the electromagnetic spectrum is observed, while a trend of fainting in optical and infrared bands is found going from low- $\alpha$ to high- $\alpha$ populations. For $1 / \varpi$, absolute magnitudes of RC stars vary between +0.12 and $-0.13 \mathrm{mag}$ from low- to high- $\alpha$ populations in the UV to MIR band. Similarly, for BJ18's method, these variations are +0.13 and -0.17 mag in the same electromagnetic region. Brightening in UV bands is an expected behavior for the older Galactic component so that this component corresponds to the high- $\alpha$ population or socalled chemically thick disk. Thick disk stars are more metalpoor than thin disk stars, causing generally higher fluxes in the UV bands of the former component. However, it was interesting to observe decreasing behavior in absolute magnitudes from optical to infrared. These findings imply that, in optic, NIR, and MIR regions, $\alpha$-elements might be contributing to the opacity in the stellar atmosphere. In short, the high- $\alpha$ AGRC stars are bluer in UV and become redder as the wavelength range of the chosen photometric band moves toward the MIR bands (see Table 3).

Based on the comparison of observational and model results given in Table 6, a similar trend of decreasing absolute magnitudes from the low- $\alpha$ to the high- $\alpha$ population is confirmed from the mock data. However, differences in absolute magnitudes for the mock catalog are around three times larger than the observational results.

Even though Gaia trigonometric parallax measurements reach down to faint magnitudes $(G=21 \mathrm{mag})$, their relative parallax errors are increasing with apparent magnitudes. Estimated distances using the photometric parallax method with absolute magnitude values of chemical population will allow more precise results than Gaia trigonometric parallax measurements of faint RC stars.

López-Corredoira et al. (2019), investigated the shape of the Galactic bulge using RC stars from the NIR Vista-VVV survey. In the study, they showed that the density distribution of RC stars toward the Bulge has two peaks. They claim that there is a 0.40 mag difference between these peaks, and the RC population cannot be represented with a single absolute magnitude. Similar behavior that is presented in this study also confirms this absolute magnitude change in RC populations. These are the signatures of the chemical evolution of the Galaxy.

Observational data of $\mathrm{RC}$ stars has a wide range of use. $\mathrm{RC}$ stars are used as probes in astronomy by measuring distances, extinction, density, age, kinematics, and chemical evolution probes of the Galaxy. This clear variation of RC stars properties with chemical composition indicates that all the relations regarding the above investigations require reconsideration.

Our overall result is that the RC absolute magnitudes are depending on chemical populations independently from metallicity 
and distance estimation methods. This study gives explicit confirmation of the chemical population dependence of RC stars.

We would like to thank the anonymous referee for his/her numerous contribution toward improving the Paper. This work has been supported by the Scientific and Technological Research Council of Turkey (TÜBİTAK), grant No: MFAG118F350. This study is partially related to Olcay Plevne's PhD thesis.

We have also made use of data from the APOGEE survey, which is part of Sloan Digital Sky Survey IV. SDSS-IV is managed by the Astrophysical Research Consortium for the Participating Institutions of the SDSS Collaboration (http:// www.sdss.org).

The GALAH survey is based on observations made at the Australian Astronomical Observatory, under programs A/ 2013B/13, A/2014A/25, A/2015A/19, A/2017A/18. We acknowledge the traditional owners of the land on which the AAT stands, the Gamilaraay people, and pay our respects to elders past and present.

This research has also made use of data from the Sloan Digital Sky Survey (SDSS DR7).

This work has made use of data from the European Space Agency (ESA) mission Gaia (https://www.cosmos.esa.int/ gaia), processed by the GaiaData Processing and Analysis Consortium (DPAC, https://www.cosmos.esa.int/web/gaia/ dpac/consortium). Funding for the DPAC has been provided by national institutions, in particular the institutions participating in the Gaia Multilateral Agreement.
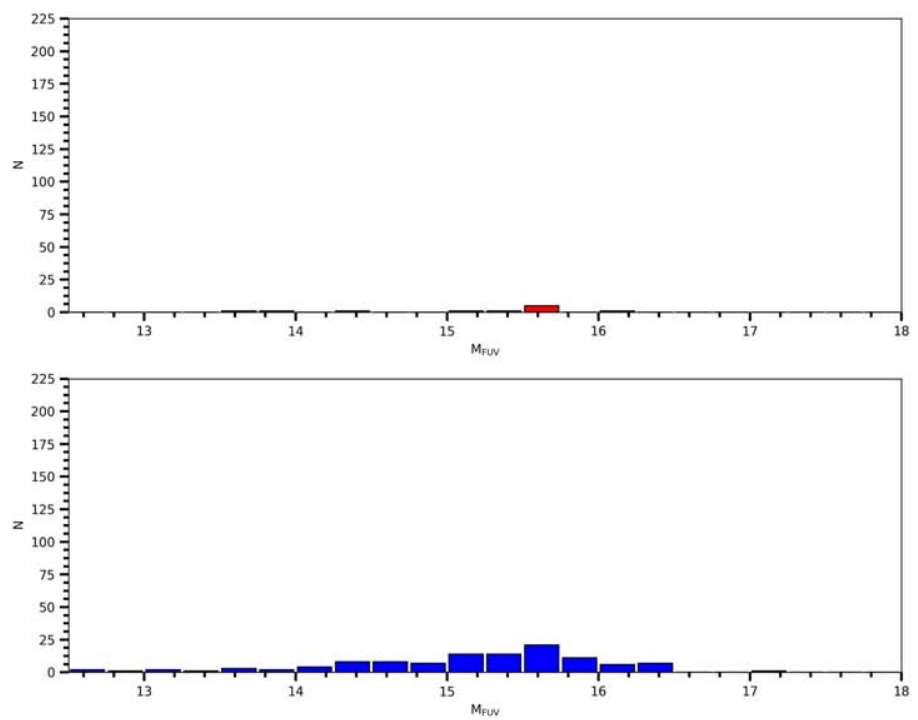

This publication makes use of data products from the Two Micron All Sky Survey, which is a joint project of the University of Massachusetts and the Infrared Processing and Analysis Center/California Institute of Technology, funded by the National Aeronautics and Space Administration and the National Science Foundation.

This publication makes use of data products from the Widefield Infrared Survey Explorer, which is a joint project of the University of California, Los Angeles, and the Jet Propulsion Laboratory/California Institute of Technology, funded by the National Aeronautics and Space Administration.

The following software and programming languages made this research possible: Python (versions $2.7 \& 3.6$ ); Astropy (version 2.0; Astropy Collaboration et al. 2013, 2018); pandas (version 0.20.2; McKinney 2011); and the VizieR catalog access tool, CDS, Strasbourg, France. The original description of the VizieR service was published in A\&AS 143, 23. For the analysis, the following software packages have been used: NumPy (van der Walt et al. 2011), matplotlib (Hunter 2007), and Jupyter Notebook (Peréz \& Granger 2007).

\section{Appendix \\ Absolute Magnitudes and Colors from UV TO MIR}

Absolute magnitude distributions of RC stars from UV to MIR bands using the $1 / \varpi$ and BJ18 methods for high- $\alpha$ (upper panels) and low- $\alpha$ (lower panels) populations are shown in Figures A1-A14.
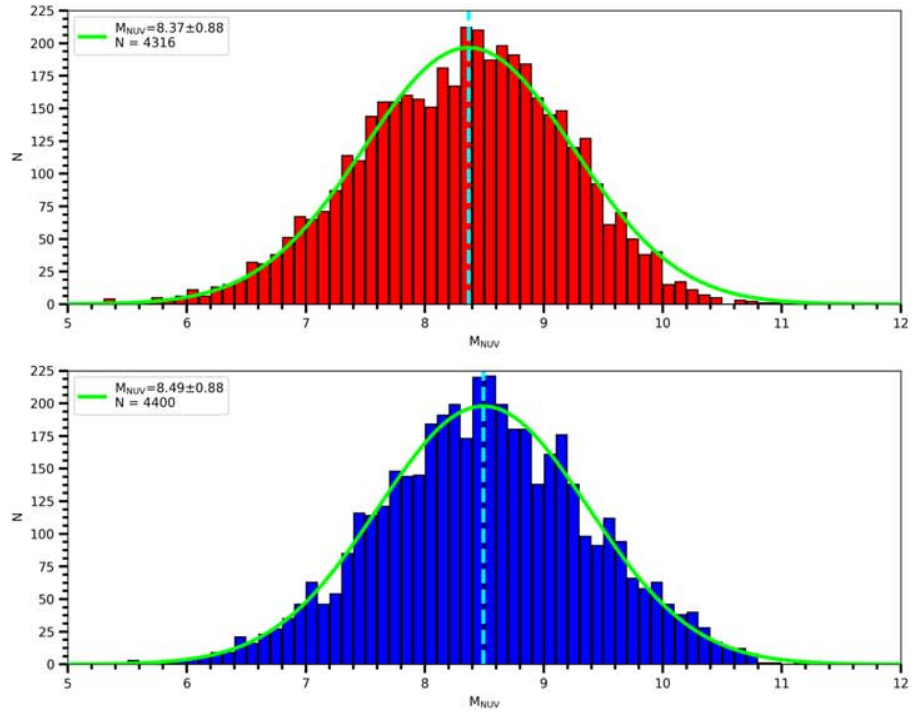

Figure A1. Absolute magnitude distribution of RC stars in GALEX NUV band that are calculated with the $1 / \varpi$ method for high- $\alpha$ (upper panel) and low- $\alpha$ (lower panel) populations. Green solid line is the Gaussian fit for the distribution and turquoise dashed line is the median value of the distribution. 

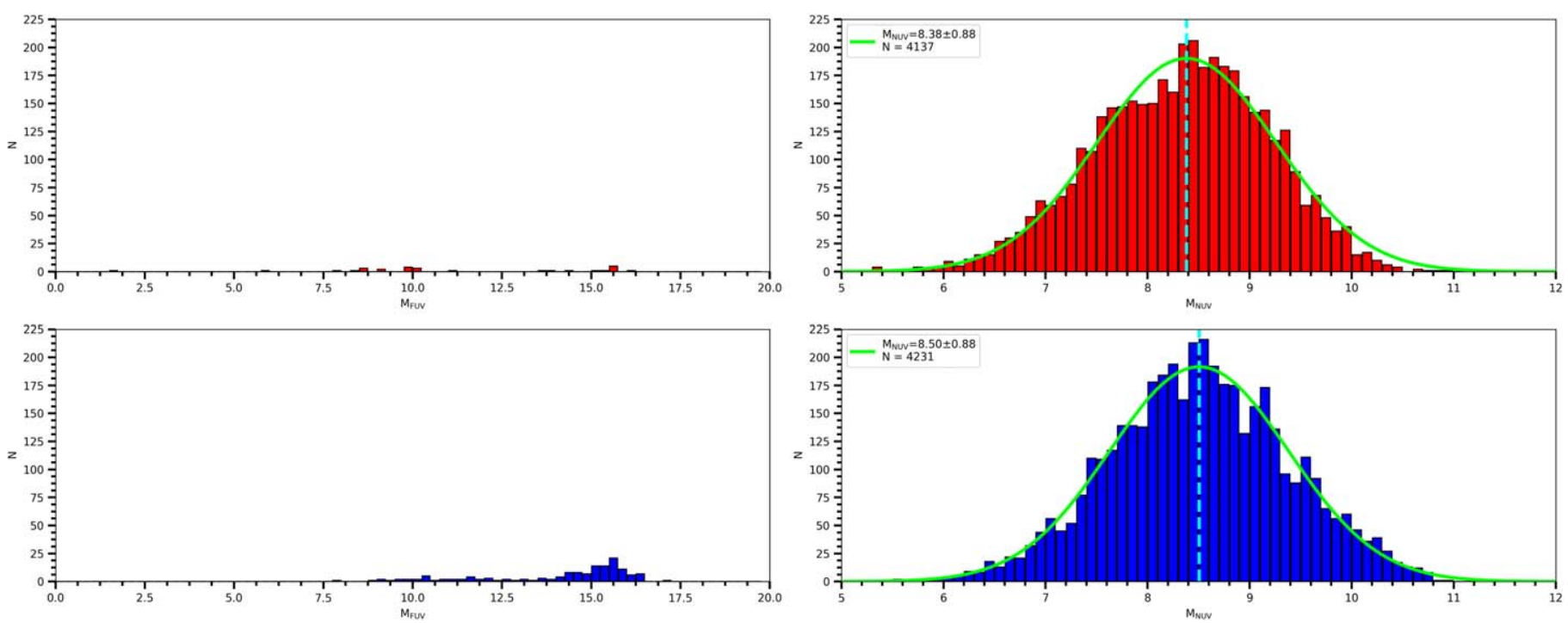

Figure A2. Absolute magnitude distribution of RC stars in GALEX NUV band that are calculated with the BJ18 method for high- $\alpha$ (upper panel) and low- $\alpha$ (lower panel) populations. Green solid line is the Gaussian fit for the distribution and turquoise dashed line is the median value of the distribution.
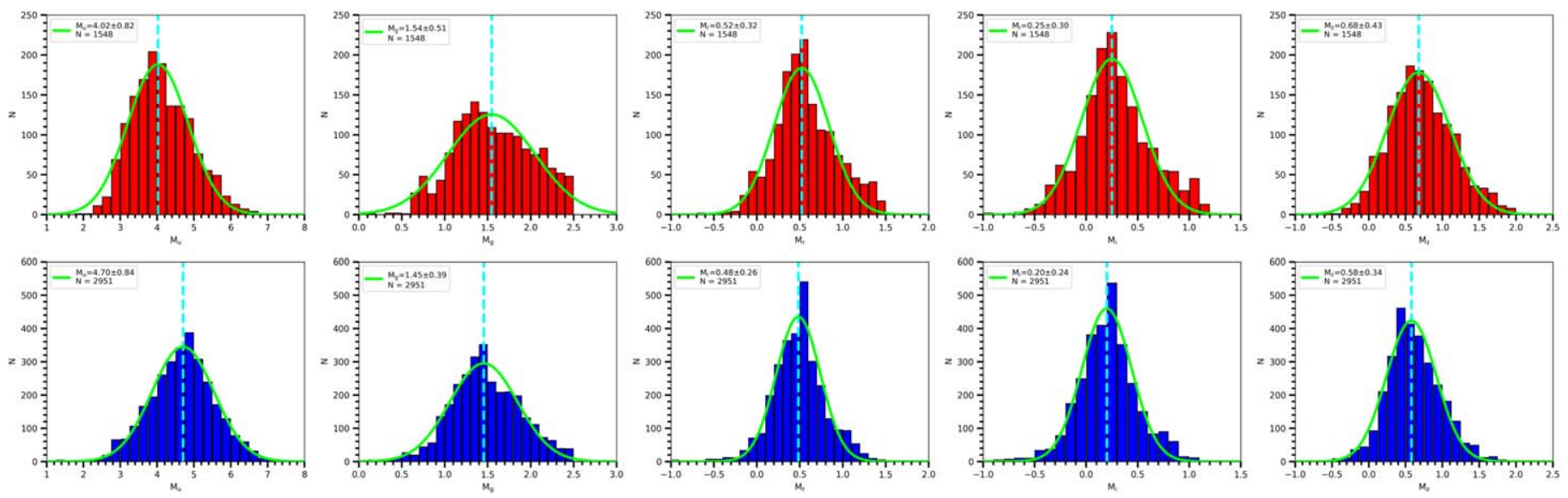

Figure A3. Absolute magnitude distribution of RC stars in SDSS $u, g, r, i$, and $z$ bands that are calculated with the $1 / \varpi$ method for high- $\alpha$ (upper panel) and low- $\alpha$ (lower panel) populations. Green solid line is the Gaussian fit for the distribution and turquoise dashed line is the median value of the distribution.
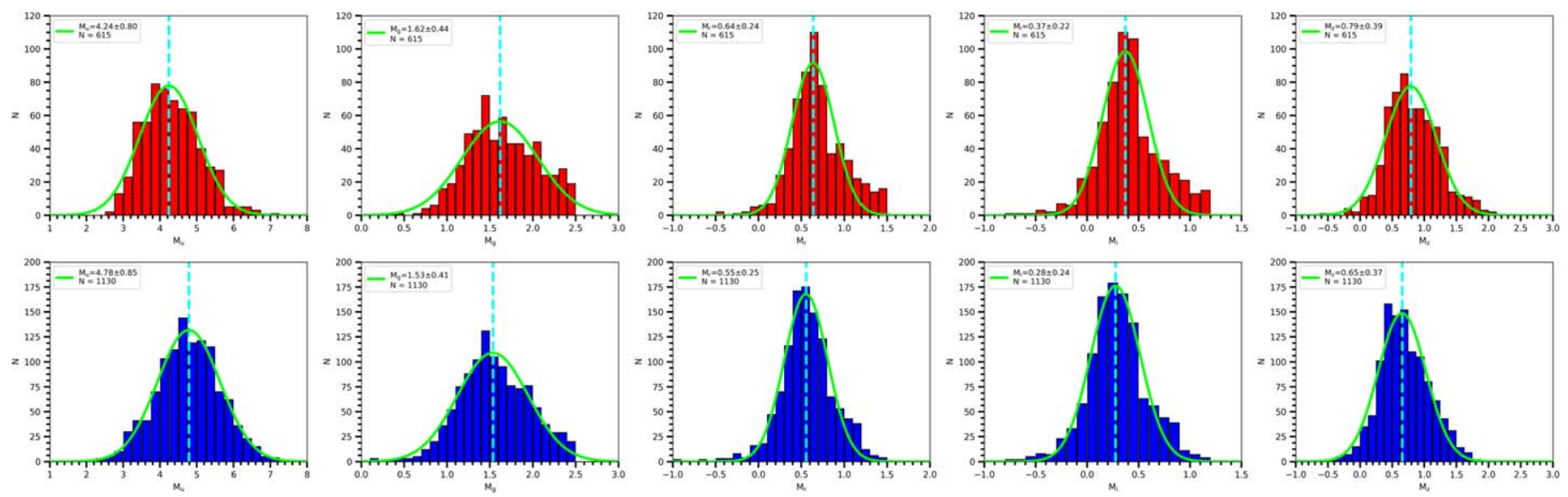

Figure A4. Absolute magnitude distribution of RC stars in SDSS $u, g, r, i$, and $z$ bands that are calculated with the BJ18 method for high- $\alpha$ (upper panel) and low- $\alpha$ (lower panel) populations. Green solid line is the Gaussian fit for the distribution and turquoise dashed line is the median value of the distribution. 

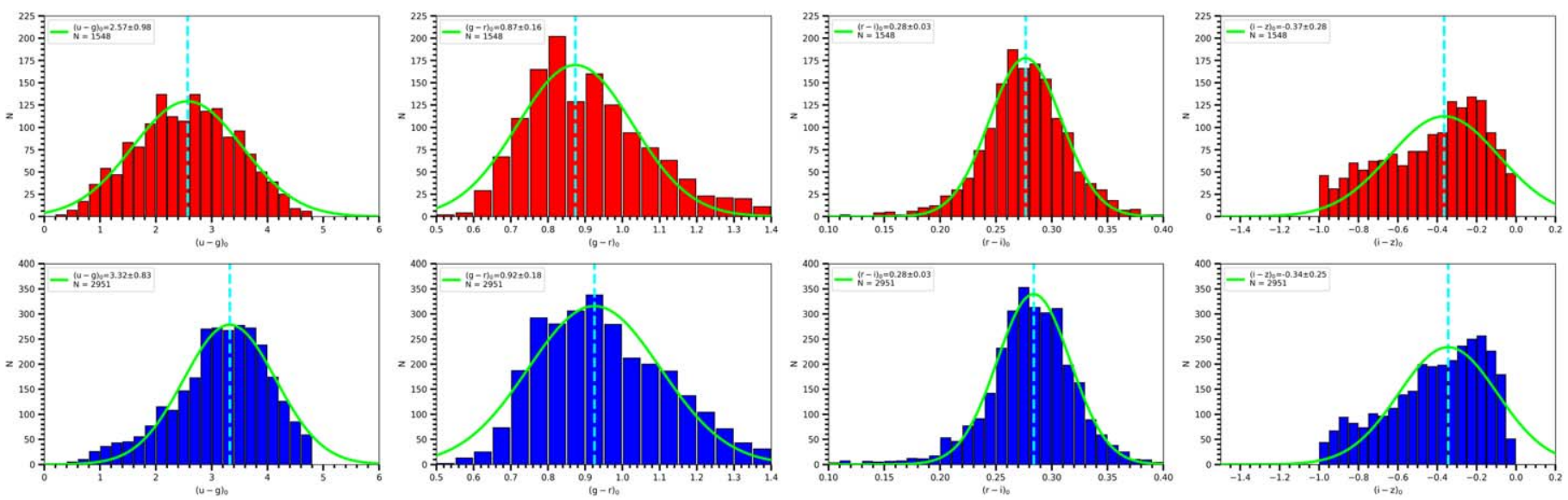

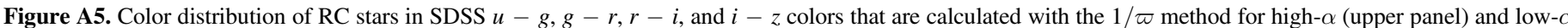
(lower panel) populations. Green solid line is the Gaussian fit for the distribution and turquoise dashed line is the median value of the distribution.
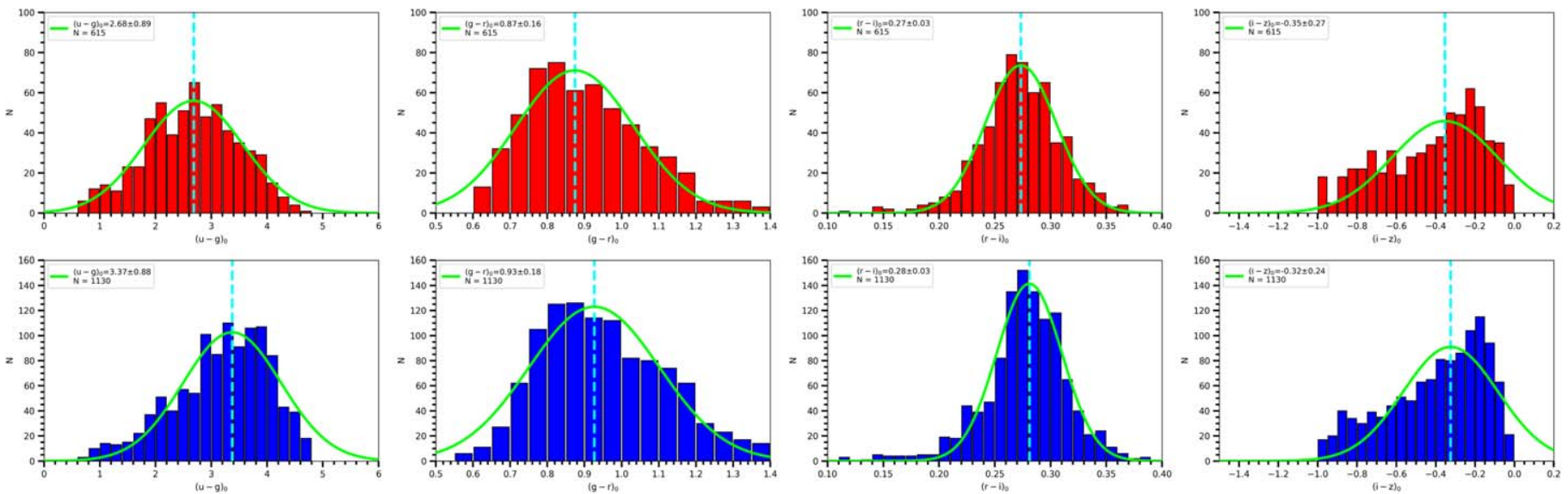

Figure A6. Color distribution of RC stars in SDSS $u-g, g-r, r-i$, and $i-z$ colors that are calculated with the BJ18 method for high- $\alpha$ (upper panel) and low- $\alpha$ (lower panel) populations. Green solid line is the Gaussian fit for the distribution and turquoise dashed line is the median value of the distribution.
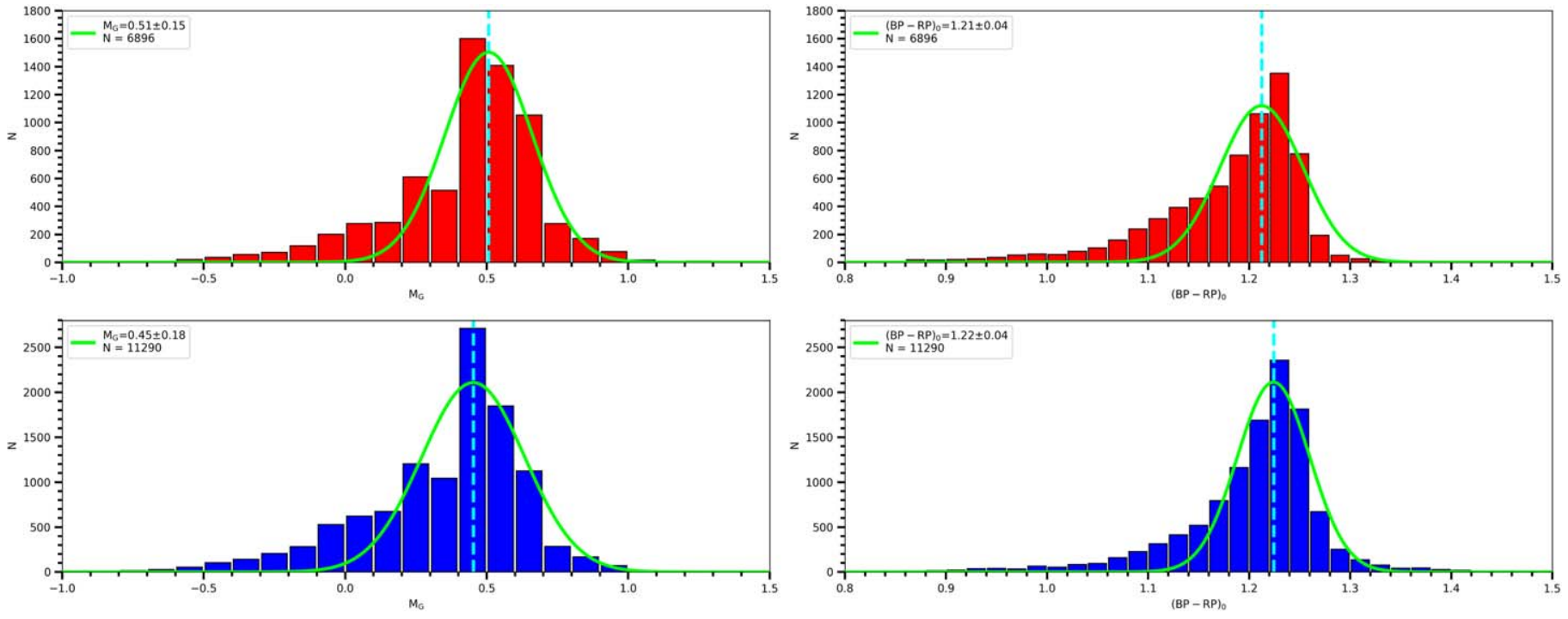

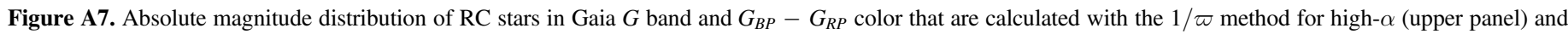
low- $\alpha$ (lower panel) populations. Green solid line is the Gaussian fit for the distribution and turquoise dashed line is the median value of the distribution. 

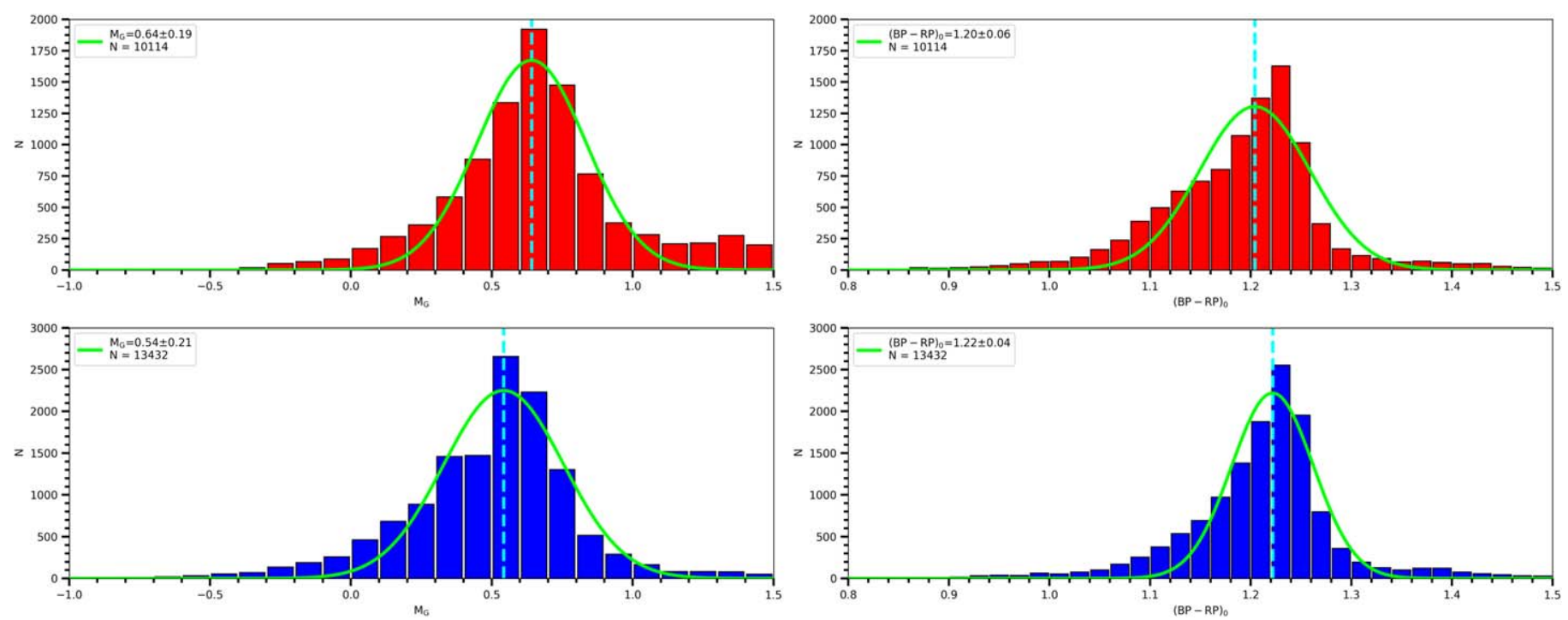

Figure A8. Absolute magnitude distribution of RC stars in Gaia $G$ band and $G_{B P}-G_{R P}$ color that are calculated with the BJ18 method for high- $\alpha$ (upper panel) and low- $\alpha$ (lower panel) populations. Green solid line is the Gaussian fit for the distribution and turquoise dashed line is the median value of the distribution.
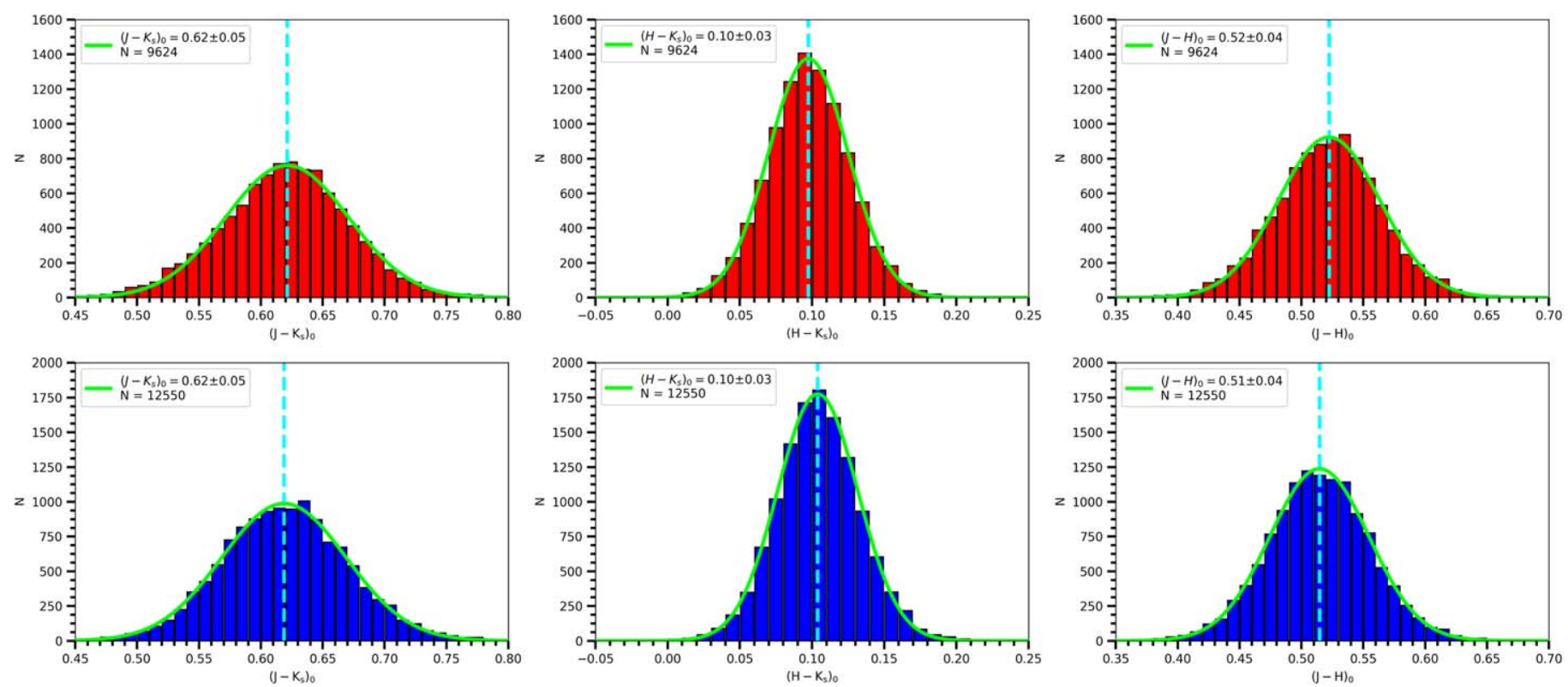

Figure A9. Color distribution of RC stars in 2MASS $J-K_{s}, H-K_{s}$, and $J-H$ colors that are calculated with the $1 / \varpi$ method for high- $\alpha$ (upper panel) and low- $\alpha$ (lower panel) populations. Green solid line is the Gaussian fit for the distribution and turquoise dashed line is the median value of the distribution. 

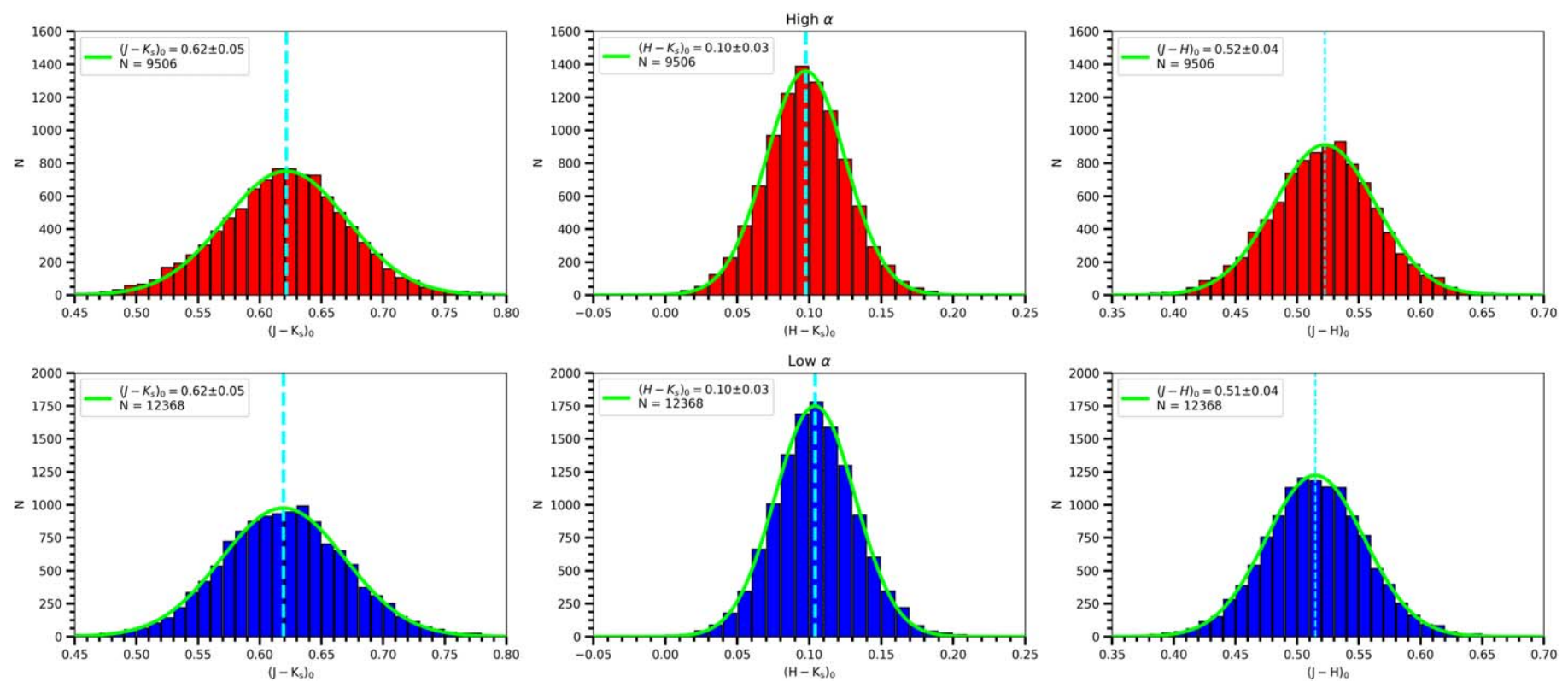

Figure A10. Color distribution of RC stars in 2MASS $J-K_{s}, H-K_{s}$, and $J-H$ colors that are calculated with the BJ18 method for high- $\alpha$ (upper panel) and low- $\alpha$ (lower panel) populations. Green solid line is the Gaussian fit for the distribution and turquoise dashed line is the median value of the distribution.
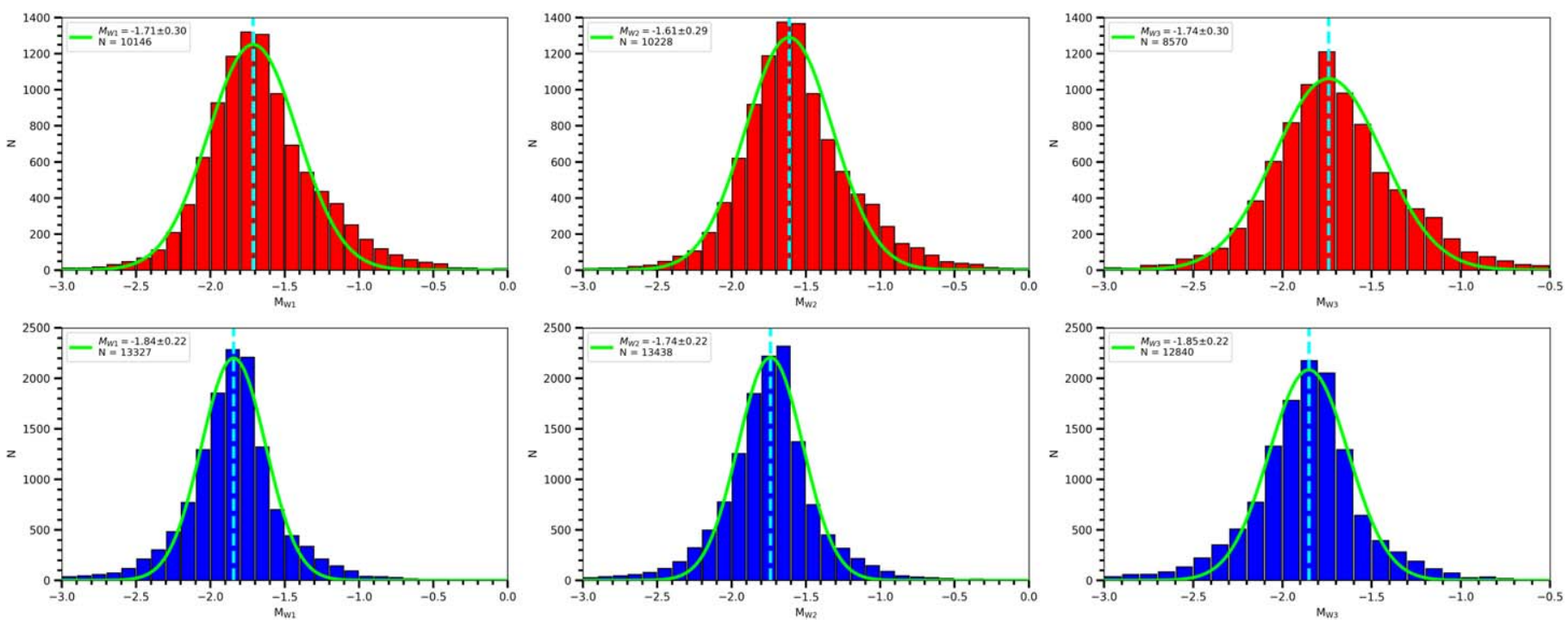

Figure A11. Absolute magnitude distribution of RC stars in $W 1, W 2, W 3$, and $W 4$ bands that are calculated with the $1 / \varpi$ method for high- $\alpha$ (upper panel) and low- $\alpha$ (lower panel) populations. 

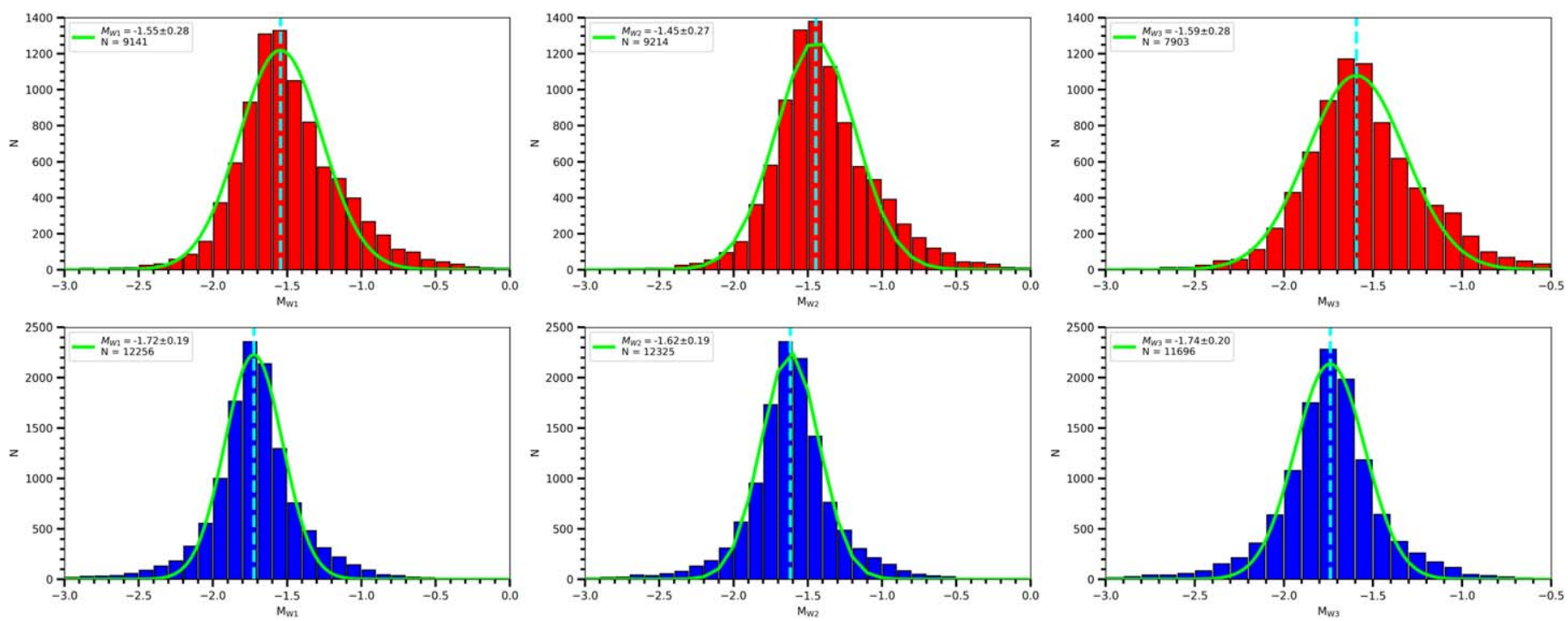

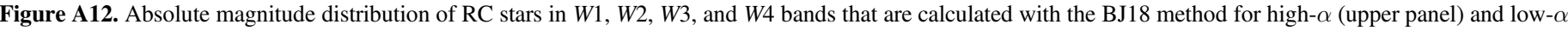
(lower panel) populations. Green solid line is the Gaussian fit for the distribution and turquoise dashed line is the median value of the distribution.
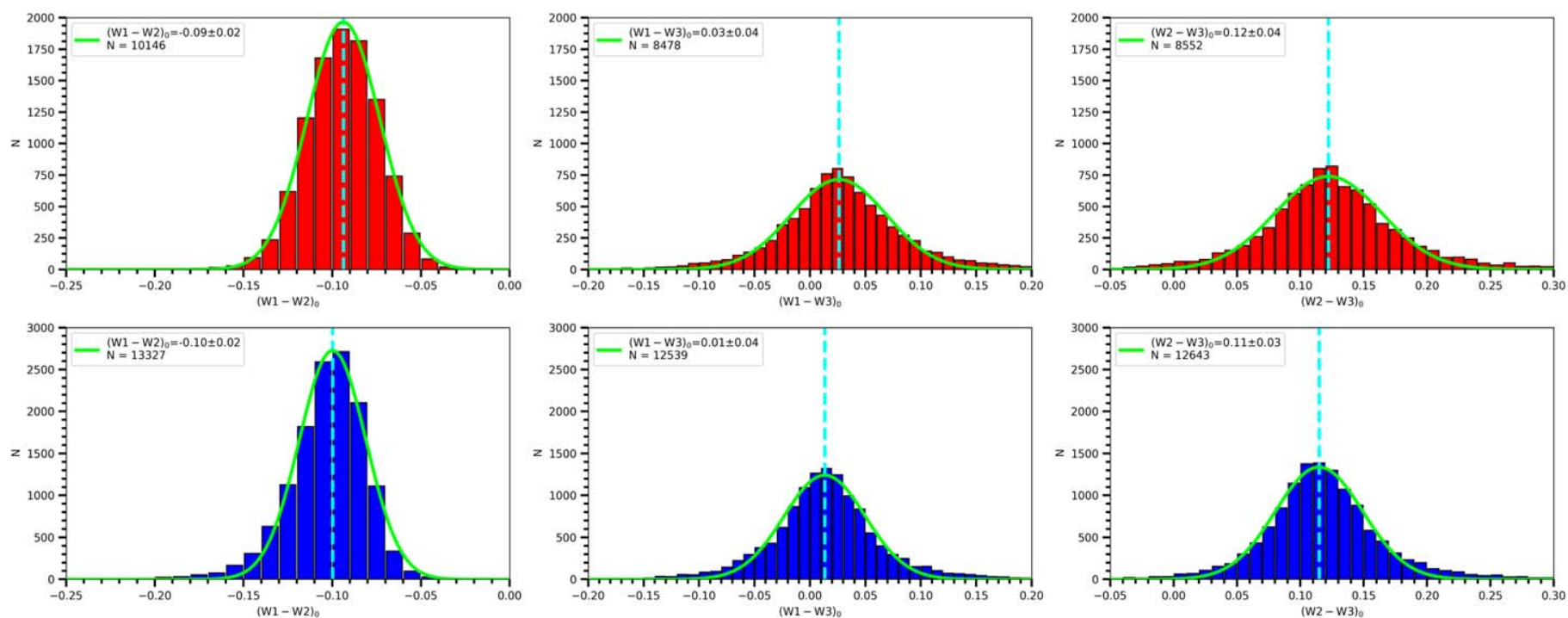

Figure A13. Color distribution of RC stars in $W 1-W 2, W 1-W 3$, and $W 2-W 3$ colors that are calculated with the $1 / \varpi$ method for high- $\alpha$ (upper panel) and low$\alpha$ (lower panel) populations. Green solid line is the Gaussian fit for the distribution and turquoise dashed line is the median value of the distribution. 

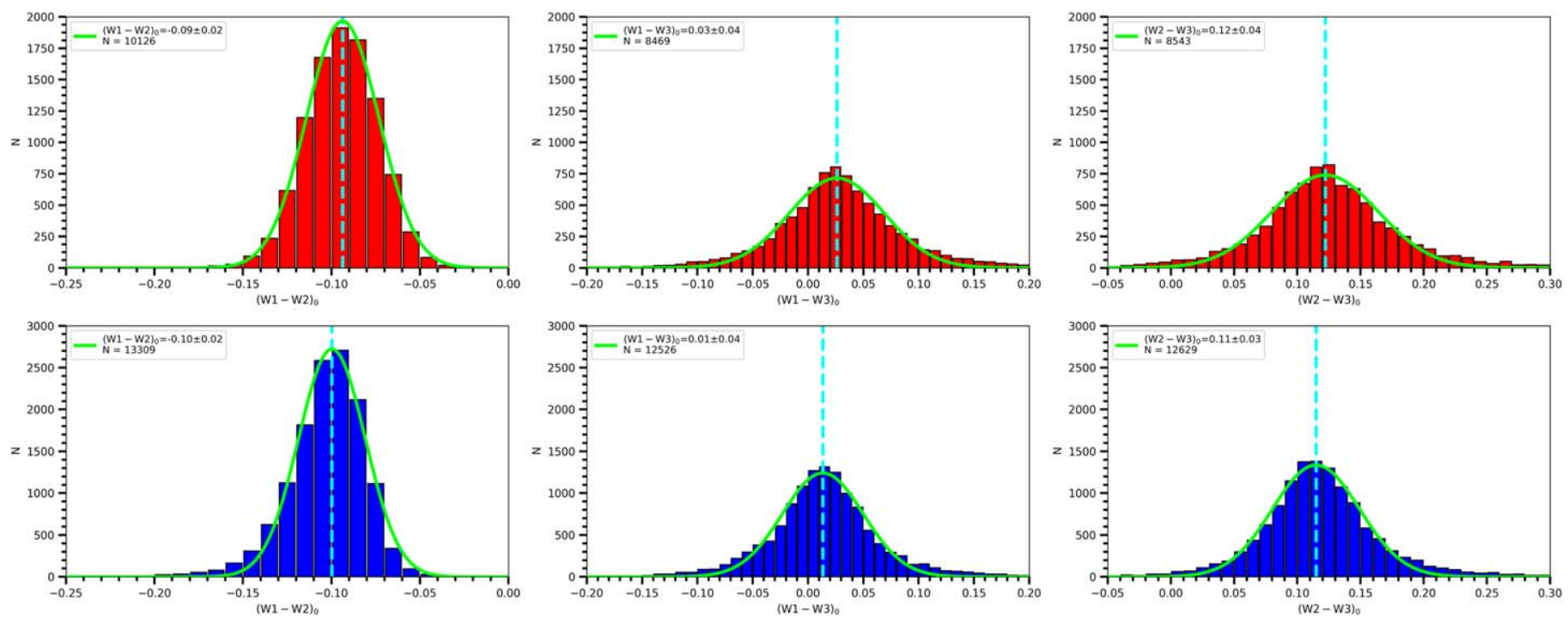

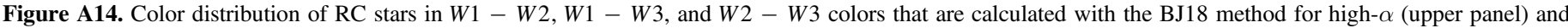
low- $\alpha$ (lower panel) populations. Green solid line is the Gaussian fit for the distribution and turquoise dashed line is the median value of the distribution.

\section{ORCID iDs}

Olcay Plevne (i) https://orcid.org/0000-0002-0435-4493

Özgecan Önal Taş (i) https://orcid.org/0000-0003-0864-1921

Selçuk Bilir (1) https://orcid.org/0000-0003-3510-1509

\section{References}

Abazajian, K. N., Adelman-McCarthy, J. K., Agüeros, M. A., et al. 2004, AJ, 128,502

Abazajian, K. N., Adelman-McCarthy, J. K., Agüeros, M. A., et al. 2009, ApJS, 182, 543

Allende Prieto, C., Majewski, S. R., Schiavon, R., et al. 2008, AN, 329, 1018 Allende Prieto, C., Majewski, S. R., Schiavon, R., et al. 2010, AN, 558, A33 Alves, D. R. 2000, ApJ, 539, 741

An, D., Pinsonneault, M. H., Masseron, T., et al. 2009, ApJ, 700, 523

Astropy Collaboration, Price-Whelan, A. M., Sipőcz, B. M., et al. 2018, AJ, 156,123

Astropy Collaboration, Robitaille, T. P., Tollerud, E. J., et al. 2013, A\&A, 558,33

Bahcall, J. N., \& Soneira, R. M. 1980, ApJS, 44, 73

Bailer-Jones, C. A. L. 2015, PASP, 127, 994

Bailer-Jones, C. A. L., Rybizki, J., Fouesneau, M., et al. 2018, AJ, 156, 58

Bensby, T., Feltzing, S., \& Lundström, I. 2003, A\&A, 410, 527

Bensby, T., Zenn, A. R., Oey, M. S., \& Feltzing, S. 2007, ApJL, 663, L13

Bianchi, L., Shiao, B., \& Thilker, D. 2017, ApJS, 230, 24

Bilir, S., Ak, T., Ak, S., Yontan, T., \& Bostanc1, Z. F. 2013a, NewA, 23, 88

Bilir, S., Cabrera-Lavers, A., Karaali, S., et al. 2008, PASA, 25, 69

Bilir, S., Karaali, S., Ak, S., et al. 2012, MNRAS, 421, 3362

Bilir, S., Karaali, S., Ak, S., Yaz, E., \& Hamzaoğlu, E. 2006, NewA, 12, 234

Bilir, S., Önal, Ö., Karaali, S., Cabrera-Lavers, A., \& Çakmak, H. 2013b, Ap\&SS, 344, 417

Bovy, J., Nidever, D. L., Rix, H., et al. 2014, ApJ, 21, 127

Bovy, J., Rix, H. W., Liu, C., et al. 2012, ApJ, 753, 148

Buder, S., Asplund, M., Duong, L., et al. 2018, MNRAS, 478, 4513

Cabrera-Lavers, A., Bilir, S., Ak, S., Yaz, E., \& López-Corredoira, M. 2007a, A\&A, 464, 565

Cabrera-Lavers, A., Hammersley, P. L., González-Fernández, C., et al. 2007b, A\&A, 465, 825

Cannon, R. D. 1970, MNRAS, 150, 111

Cannon, R. D., \& Lloyd, C. 1969, MNRAS, 144, 449

CCelebi, M., Bilir, S., Ak, S., et al. 2019, Ap\&SS, 364, 172

Chen, Y. Q., Casagrande, L., Zhao, G., et al. 2017, ApJ, 480, 2

Chiappini, C., Matteucci, F., \& Gratton, R. 1997, ApJ, 477, 765

Coşkunoğlu, B., Ak, S., Bilir, S., et al. 2012, MNRAS, 419, 2844

Cutri, R. M., Skrutskie, R. M., van Dyk, S., et al. 2003, yCat, 2246, 0

Cutri, R. M., et al. 2013, yCat, 2328, 0 de Silva, G. M., Freeman, K. C., Bland-Hawthorn, J., et al. 2016, MNRAS, 449, 2604

ESA 1997, yCat, 1239, 0

Fan, X. 1999, AJ, 117, 2528

Faulkner, J. 1966, ApJ, 144, 995

Fiorucci, M., \& Munari, U. 2003, A\&A, 401, 781

Francis, C., \& Anderson, E. 2014, MNRAS, 441, 1105

Freeman, K. C., \& Bland-Hawthorn, J. 2002, ARA\&A, 40, 487

Fuhrmann, K. 1998, A\&A, 338, 161

Fuhrmann, K. 2008, MNRAS, 384, 17

Fukugita, M., Ichikawa, T., Gunn, J. E., et al. 1996, AJ, 111, 1748

Gaia Collaboration, Brown, A. G. A., Vallenari, A., et al. 2018, A\&A, 616, A1

Gaia Collaboration, Prusti, T., de Bruijne, J. H. J., et al. 2016, A\&A, 595, A1

Gilmore, G., \& Wyse, R. F. G. 1985, AJ, 90, 2015

Girardi, L. 2016, ARA\&A, 54, 95

Girardi, L., Groenewegen, M. A. T., Weiss, A., \& Salaris, M. 1998, MNRAS, 301,149

Girardi, L., \& Salaris, M. 2001, MNRAS, 323, 109

Gratton, R., Carretta, E., Matteucci, F., \& Sneden, C. 1996, in ASP Conf. Ser. 92, Formation of the Galactic Halo, ed. H. L. Morrison \& A. Sarajedini (San Francisco, CA: ASP), 307

Grocholski, A. J., \& Sarajedini, A. 2002, AJ, 123, 1603

Groenewegen, M. A. T. 2008, A\&A, 488, 935

Hawkins, K., Leistedt, B., Bovy, J., \& Hogg, D. W. 2017, MNRAS, 471, 722

Haywood, M. 2008, MNRAS, 288, 1175

Haywood, M., Snaith, O., Lehnert, M. D., et al. 2019, A\&A, 625, A105

Holtzman, J. A., Hasselquist, S., Shetrone, M., et al. 2018, AJ, 156, 125

Hunter, J. D. 2007, CSE, 9, 90

Iben, I. J. 1967, ApJ, 147, 624

Karaali, S., Bilir, S., \& Yaz Gökçe, E. 2013, Ap\&SS, 346, 89

Karaali, S., Bilir, S., Yaz Gökçe, E., \& Plevne, O. 2019, PASA, 36, 40

Laney, C. D., Joner, M. D., \& Pietrzyński, G. 2012, MNRAS, 419, 1637

López -Corredoira, M., Lee, Y. W., Garzón, F., et al. 2019, A\&A, 627, a3

Lutz, T. E., \& Kelker, D. H. 1973, PASP, 85, 573

Majewski, S. R. 1993, ARA\&A, 31, 575

Majewski, S. R., Schiavon, R. P., Frinchaboy, P. M., et al. 2017, ApJ, 154, 94

Marshall, D. J., Robin, A. C., Reyle, C., et al. 2006, A\&A, 453, 635

Martig, M., Fouesneau, M., Rix, H. W., et al. 2016, MNRAS, 406, 3655

Martin, D. C., Fanson, J., Schiminovich, D., et al. 2005, ApJL, 619, 1

McKinney, W. 2011, 14, Python for High Performance and Scientific Computing

Michalik, D., Lindegren, L., \& Hobbs, D. 2015, A\&A, 574, A115

Mohammed, S., Schiminovich, D., Hawkins, K., et al. 2019, ApJ, 872, 95

Navarro, J. F., Abadi, M. G., Venn, K. A., Freeman, K. C., \& Anguiano, B. 2011, MNRAS, 412, 1203

Ness, M., Hogg, D. W., Rix, H. W., Ho, A. Y. Q., \& Zasowski, G. 2015, ApJ, 808,16

Olivares, J., Bouy, H., Sarro, L. M., et al. 2019, A\&A, 625, A115 
Önal Taş, Ö., Bilir, S., \& Plevne, O. 2018, Ap\&SS, 363, 35

Önal Taş, Ö., Bilir, S., Seabroke, G. M., et al. 2016, PASA, 33, e044

Oudmaijer, R. D., Groenewegen, M. A. T., \& Shrjiver, H. 2002, MNRAS, 294, L41

Paczyński, B., \& Stanek, K. Z. 1998, ApJL, 494, L219

Pedregosa, F., Varoquaux, G., Gramfort, A., et al. 2011, Journal of Machine Learning Research, 12, 2830

Pérez, F., \& Granger, B. E. 2007, CSE, 9, 21

Perryman, M. A. C., Brown, A. G. A., Lebreton, Y., et al. 1998, A\&A, 331, 81 Perryman, M. A. C., Lindegren, L., Kovalevsky, J., et al. 1997, A\&A, 323, L49

Plevne, O., Ak, T., Karaali, S., et al. 2015, PASA, 32, e043

Prochaska, J. X., Naumov, S. O., Carney, B. W., McWilliam, A., \& Wolfe, A. M. 2000, AJ, 120, 2513

Reddy, B. E., Lambert, D. L., \& Allende Prieto, C. 2006, MNRAS, 367, 1329

Robin, A. C., Marshall, D. J., Schultheis, M., et al. 2012, A\&A, 538, A106

Robin, A. C., Reylé, C., Derrière, S., et al. 2003, A\&A, 409, 523

Ruiz-Dern, L., Babusiaux, C., Arenou, F., et al. 2018, A\&A, 609, A116

Saguner, T., Munari, U., Fiorucci, M., \& Vallenari, A. 2011, A\&A, 540, A10
Salaris, M. 2013, in IAU Symp. 289, Advancing the Physics of Cosmic Distances, ed. R. de Grijs (Cambridge: Cambridge Univ. Press), 145

Salaris, M., \& Girardi, L. 2002, MNRAS, 337, 332

Sarajedini, A. 1999, AJ, 118, 2321

Schlafly, E. F., \& Finkbeiner, D. P. 2011, ApJ, 737, 103

Skrutskie, M. F., Cutri, R. M., Stiening, R., et al. 2006, AJ, 131, 1163

Smith, H., Jr. 1987, A\&A, 171, 336

Tunçel Güçtekin, S., Bilir, S., Karaali, S., et al. 2016, Ap\&SS, 361, 186

Tunçel Güçtekin, S., Bilir, S., Karaali, S., et al. 2019, AdSpR, 63, 1360

Twarog, B. A., Anthony-Twarog, B. J., \& Bricker, A. R. 1999, AJ, 117, 1816

Udalski, A. 2000, ApJL, 531, L25

van der Walt, S., Colbert, S. C., \& Varoquaux, G. 2011, CSE, 13, 22

van Helshoecht, V., \& Groenewegen, M. A. T. 2007, A\&A, 463, 559

van Leeuwen, F. 2007, A\&A, 474, 653

Wang, S., \& Chen, X. 2019, ApJ, 877, 116

Wright, E. L., Eisenhart, P. R. M., Mainzer, A. K., et al. 2010, AJ, 140, 1868 Wyse, R. F. G., \& Gilmore, G. 1988, AJ, 95, 1404

Yaz Gökçe, E., Bilir, S., Öztürkmen, N. D., et al. 2013, NewA, 25, 19

Yuan, H. B., Liu, X. W., \& Xiang, M. S. 2013, MNRAS, 430, 2188 